\title{
Rigid upper bounds for the angular momentum and centre of mass of non-singular asymptotically anti-de Sitter space-times
}

\author{
Piotr T. Chruściel* \\ LMPT, Fédération Denis Poisson, Tours \\ and Albert Einstein Institute, Golm ${ }^{\dagger}$ \\ Daniel Maerten \\ Institut de Mathématiques et de Modélisation de Montpellier (I3M) \\ Université Montpellier II \\ Paul Tod $\operatorname{To}^{\S}$ \\ Mathematical Institute and St John's College \\ Oxford
}

July 27, 2018

\begin{abstract}
We prove upper bounds on angular momentum and centre of mass in terms of the Hamiltonian mass and cosmological constant for non-singular asymptotically anti-de Sitter initial data sets on spin manifolds satisfying the dominant energy condition. We work in space-dimensions larger than or equal to three, and allow a large class of asymptotic backgrounds, with spherical and non-spherical conformal infinities; in the latter case, a spinstructure compatibility condition is imposed. We give classes of non-trivial examples saturating the inequality. We analyse the borderline case in space-time dimension four: for spherical cross-sections of Scri, equality together with completeness occurs only in anti-de Sitter space-time. On the other hand, in the toroidal case, regular non-trivial initial data sets saturating the bound exist.
\end{abstract}

\section{Introduction}

In recent work [35] one of us (DM) proved an inequality satisfied by the global charges for three-dimensional asymptotically anti-de Sitter initial data sets with

\footnotetext{
${ }^{*}$ E-mail Piotr.Chrusciel@lmpt.univ-tours.fr, URL www.phys.univ-tours.fr/ piotr

$\dagger$ Visiting fellow.

¥ E-mail: maerten@math.univ-montp2.fr

$\S$ E-mail: paul.tod@st-johns.oxford.ac.uk
} 
spherical conformal infinity. In this paper we extend that work in several directions by a consideration of more general initial data sets $(\mathscr{S}, g, K)$ on a spin manifold $\mathscr{S}$. The extensions are as follows:

- We prove the corresponding inequalities in dimensions $n+1, n \geq 3$, with a spin-structure condition for non-spherical Scris (Theorem 2.1).

- For spherical Scris we obtain optimal inequalities for $n$ equal to four and five, as well as some natural but non-optimal inequalities for all $n \geq 3$ (Theorem 3.1; by optimal we mean that saturation of the inequality is a necessary and sufficient condition for the existence of space-time Killing spinors at $\mathscr{S}$ ). For $n=3$, we show that the total momentum cannot be null and give a sufficient condition slightly stronger than equality for the data to be anti-de Sitter (Theorem 3.9). Still for $n=3$, when the associated space-time has a complete Scri with spherical cross-sections, we prove that equality happens only in anti-de Sitter space-time (Section 3.4).

- For toroidal Scris we obtain optimal inequalities for all $n \geq 3$ (Section 4.1), and we point out the existence of large families of non-singular (nonvacuum) initial data sets which saturate the inequality (Sections 4.3 and 4.4).

- We prove that, in dimension $3+1$, with spherical or toroidal Scri, black hole solutions saturating the inequality do not exist (Section 3.2).

- We obtain an angular-momentum bound for general conformal boundaries at infinity with covariantly constant spinors, again under a spin-structure condition (Section 4.2).

\section{Global charges and their positivity}

In this work we consider $n$-dimensional general relativistic data sets $(\mathscr{S}, g, K)$, which are asymptotically anti-de Sitter (adS) in the following sense: First, we assume that there exists a Riemannian background metric $b$ which, in the asymptotic region, is of the form

$$
b=d r^{2}+\stackrel{\circ}{f}(r) \check{h},
$$

where $\check{h}$ is either a unit round metric on $S^{n-1}$ (then with the cosmological constant $\Lambda$ normalised to $-n$, and up to change of origin in $\left.r, \stackrel{\circ}{f}(r)=\sinh ^{2} r\right)$, or $\check{h}$ is a Ricci flat metric on an $(n-1)$-dimensional compact manifold ${ }^{n-1} M$ (then, again up to these choices, $f(r)=e^{2 r}$ ), where the space-dimension $n$ is greater than or equal to 3 . By [10], with those $f$, the initial data $(\mathscr{S}, b, 0)$ arise from static solutions of vacuum Einstein equations with a negative cosmological constant $^{1}$. Note that in the spherical case, or if $\left({ }^{n-1} M, \breve{h}\right)$ is a flat torus $T^{n-1}$,

\footnotetext{
${ }^{1}$ It might seem natural also to allow $\left({ }^{n-1} M, \breve{h}\right)$ to be a negatively curved Einstein manifold [10]. However, we shall see shortly that such solutions do not seem to fit into a Witten-type positivity argument, which is the main concern of this work.
} 
then $(\mathscr{S}, b, 0)$ are initial data for anti-de Sitter space-time, or a quotient thereof. In all cases $(\mathscr{S}, b, 0)$ provide initial data for a static Einstein metric.

Next, there is a well-established set of decay conditions which guarantee finite and well defined global charges, see [14, 16], compare [25, 41, 42]. Following these works, we shall assume that there exist constants $k \geq 1, \alpha>n / 2$ and $C>0$ such that for large $r$ we have ${ }^{2}$

$$
|g-b|_{b}+|\stackrel{\circ}{D} g|_{b}+\cdots+|\underbrace{\stackrel{\circ}{D} \cdots \stackrel{\circ}{D}}_{k \text { factors }} g|_{b}+|K|_{b}+\cdots+|\underbrace{\stackrel{\circ}{D} \cdots \stackrel{\circ}{D}}_{k-1 \text { factors }} K|_{b} \leq C e^{-\alpha r} .
$$

Here $|\cdot|_{b}$ denotes the norm of a tensor field with respect to the metric $b$, and $\stackrel{\circ}{D}$ is the covariant derivative of $b$. These decay conditions have been chosen because of simplicity of the analysis involved; it should be recognised that they are restrictive, and a completely satisfactory treatment should allow weaker boundary conditions, compare [15] for a related analysis in the context of a vanishing cosmological constant $\Lambda=0$.

To define the global charges, let $X$ be a Killing vector in the asymptotic region of the associated background space-time. It is well known that each such $X$ defines a Hamiltonian associated with the flow along $X[13,15,16,25]$, as follows: Let $V$ be the normal component of $X$ with respect to the spacetime background metric, and let $Y$ be the tangential component thereof; when defined along a spacelike hypersurface, such pairs $(V, Y)$ are called Killing Initial Data (KIDs). Then the Hamiltonian $H(V, Y)$ corresponding to $X$ (which we identify with the pair $(V, Y))$ takes the form:

$$
H(V, Y):=\lim _{R \rightarrow \infty} \frac{1}{16 \pi} \int_{r=R}\left(\mathbb{U}^{i}(V)+\mathbb{V}^{i}(Y)\right) d S_{i},
$$

where

$$
\begin{gathered}
\mathbb{U}^{i}(V):=2 \sqrt{\operatorname{det} g}\left(V g^{i[k} g^{j] l} \stackrel{\circ}{D}_{j} g_{k l}+D^{[i} V g^{j] k}\left(g_{j k}-b_{j k}\right)\right. \\
\mathbb{V}^{i}(Y):=2 \sqrt{\operatorname{det} g}\left(K^{i}{ }_{j}-K_{k}^{k} \delta_{j}^{i}\right) Y^{j}
\end{gathered}
$$

Here all indices are space indices, running from 1 to $n$, and $\stackrel{\circ}{D}$ is the Levi-Civita derivative of the space background metric $b$.

The normalisation constant $1 / 16 \pi$ in $(2.2)$ is convenient in dimension $3+1$ when $\mathscr{I}$ has spherical cross-section, but rather arbitrary in higher dimensions, or when non-spherical cross-sections are considered.

We shall give conditions under which a Witten-type proof of positivity of global charges applies. By this we understand an identity for a spinor $\psi$, relating an appropriate component of the global charges to an integral over $\mathscr{S}$ of a bilinear in $\psi$; the bilinear is positive given a positivity hypothesis on the energymomentum of the initial data set and a differential equation for $\psi$ at $\mathscr{S}$, with suitable asymptotic conditions for $\psi$ on $\mathscr{S}$; positivity of the relevant component

\footnotetext{
${ }^{2}$ In many of our arguments it is sufficient to assume the weaker, integral-type, decay conditions of [15, Section 3], but we have not checked whether all the calculations go through under such conditions.
} 
of the global charge follows from a suitable existence theorem for this differential equation; in the asymptotically-adS setting, we require $\psi$ to be asymptotic to an imaginary Killing spinor, a notion which we define below. (For more details of the Witten argument, giving the spinor identity and an existence theorem in this setting, see e.g. $[14,15,35,41]$. Examples in which the Witten argument does not apply are given in $[18,28]$.)

Thus the Witten-type proof needs a positivity hypothesis on the energymomentum of the initial data set, which will be the dominant energy condition or DEC. Denoting the cosmological constant by $\Lambda$, we set

$$
\rho:=R-|K|^{2}+\left|\operatorname{tr}_{g} K\right|^{2}-2 \Lambda, \quad J^{i}=D_{j} K^{i}{ }_{j}-D^{i} K^{j}{ }_{j},
$$

where $R$ is the scalar curvature of $g$. The DEC reads then

$$
\rho \geq|J|_{g} .
$$

Next, we need an imaginary Killing spinor for the background metric b; by definition, this is a spinor field $\psi$ in the asymptotic region $\mathscr{S}_{\text {ext }}:=\left[R_{0}, \infty\right) \times$ ${ }^{n-1} M$ solving the set of equations

$$
\forall X \in T \mathscr{S} \quad \stackrel{\circ}{D}_{X} \psi=-i \sqrt{\frac{-\Lambda}{2 n(n-1)}} X \cdot \psi,
$$

where $X$. denotes the Clifford product of $X$, and $\stackrel{\circ}{D}$ is the usual Riemannian spinorial connection associated with the metric $b$. Such spinor fields are known to exist when ${ }^{n-1} M$ is a sphere; we point out several (well known $[8,11,23,31$, 34]) further examples, with alternative topologies at infinity, in Section 4 below. The field $\psi$ is a section of a bundle of spinors ${ }^{3}$ which we will denote by $\mathfrak{S}^{\prime}$.

(It is worthwhile pointing out at this stage some more cases when the Witten-type argument cannot be carried through: manifolds $\mathscr{S}_{\text {ext }}$, with $\left({ }^{n-1} M, \breve{h}\right)$ having negative Ricci curvature, do not admit imaginary Killing spinors. This can be seen as follows: first, any imaginary Killing spinor leads to a Killing vector in $\mathscr{S}_{\text {ext }}$. But it is known, e.g. from the analysis in [2, Appendix A], that there are no Killing vectors on $\mathscr{S}_{\text {ext }}$ in this case. Thus, no lower bounds on the mass can be obtained by Witten-type techniques when, e.g., ${ }^{n-1} M$ is a two-dimensional higher genus surface.)

For the Witten argument to go through, we need to assume that $\mathscr{S}$ admits a spin structure. Note that the spinor field $\psi$ already singles out a spin structure on $\mathscr{S}_{\text {ext }}$, which is necessarily compatible with the one of $\mathscr{S}$ when ${ }^{n-1} M$ is simply connected. However, those spin structures might be incompatible when ${ }^{n-1} M$ is not simply connected. A key, rather restrictive, hypothesis in our work is that

the bundle $\mathfrak{S}^{\prime}$ over $\mathscr{S}_{\text {ext }}$ extends to a bundle of spinors $\mathfrak{S}$ over $\mathscr{S}$.

\footnotetext{
${ }^{3}$ By a "spinor field" we mean a section of a Hermitian bundle associated to the Spin principal bundle over $\mathscr{S}$, equipped with an action of the Clifford algebra of $\mathscr{S}$ via anti-Hermitian bundle-morphisms. In what follows we shall freely make use of "doubling constructions" such as the one in (2.11) below, and therefore we do not impose the often-implicitly-used condition that the representation of the Clifford algebra carried by the spinor bundle is irreducible.
} 
A short discussion of the hypothesis (2.7) is in order. First, (2.7) is satisfied by all product topologies $\mathscr{S}=\mathbb{R} \times{ }^{n-1} M$, or $\mathscr{S}=[0, \infty) \times{ }^{n-1} M$. These examples include the hyperbolic-cusp solutions (4.1) below, or the Kottler black holes [32] with toroidal topology at infinity. On the other hand, (2.7) is not satisfied by the Horowitz-Myers solutions [28]. Now, in that last example $\mathscr{S}$ is the union of a compact set and of the asymptotic region $\mathscr{S}_{\text {ext }}$, and in such a context we have the following ${ }^{4}$ : If ${ }^{n-1} M=\mathbb{T}^{2}$, the two-dimensional torus, and $\mathscr{S}$ has no boundary (other than the conformal boundary at infinity), then the trivial spin structure on $\mathbb{T}^{2}$, which does admit parallel spinors, never extends [33, p. 91] when compactness of the conformal completion of $\mathscr{S}$ is imposed. On the other hand, for all higher-dimensional toroidal boundaries at infinity ${ }^{n-1} M=\mathbb{T}^{n-1}$, $n \geq 4$, compact boundaryless fillings for the trivial spin structure of $\mathbb{T}^{n-1}$ exist $[33$, p. 92]. All this leads to a large class of examples where (2.7) holds.

For the analytical arguments to go through, we need further to assume that $(\mathscr{S}, g)$ is complete, either without boundary, or with a compact boundary satisfying the following: Let $\lambda$ be the extrinsic curvature tensor of $\partial \mathscr{S}$ (considered as a submanifold of $\mathscr{S}$, recall that there is no space-time involved at this stage) with respect to an inward-pointing unit normal $\nu$, let $h$ be the metric induced on $\partial \mathscr{S}$ by $g$. The boundary contribution which arises in the Witten argument with a spinor field satisfying the boundary condition of [21] (compare [26]) will have the favorable sign provided that the boundary is either weakly future trapped,

$$
\operatorname{tr}_{h} \lambda+h^{a b} K_{a b} \leq 0,
$$

or weakly past trapped, which corresponds to changing the sign in front of the $K$ term in (2.8). An alternative condition which allows one to conclude is that considered in $[14,35]$. Setting $k(\nu)=K_{i a} \nu^{i} d x^{a}$, where the $x^{a}$ 's are coordinates on $\partial \mathscr{S}$, we then assume that

$$
\operatorname{tr}_{h} \lambda+|k(\nu)|_{h} \leq \sqrt{\frac{-2(n-1) \Lambda}{n}}
$$

(see [14, Remark 4.8] for a discussion of $(2.9)$ when $k(\nu)=0$ ).

Without loss of generality [33], we can assume that the spinor bundle $\mathfrak{S}$ is equipped with a Hermitian product $\langle\cdot, \cdot\rangle$ such that Clifford multiplication by vectors tangent to $\mathscr{S}$ is an anti-Hermitian endomorphism. In the construction we will need a bundle isomorphism $\gamma^{0}: \mathfrak{S} \rightarrow \mathfrak{S}$ with the following properties:

$$
\begin{aligned}
& \left(\gamma^{0}\right)^{2}=1, \\
& \forall X \in T \mathscr{S} \quad \gamma^{0} X \cdot=-X \cdot \gamma^{0} \\
& \left(\gamma^{0}\right)^{\dagger}=\gamma^{0} \\
& D \gamma^{0}=\gamma^{0} D
\end{aligned}
$$

where $\left(\gamma^{0}\right)^{\dagger}$ denotes the conjugate of $\gamma^{0}$ with respect to the Hermitian product $\langle\cdot, \cdot\rangle$ (by a small abuse of notation, we shall use this symbol for the inner product

\footnotetext{
${ }^{4}$ We are grateful to M. Stern for discussions and references concerning the compatibility of spin structures.
} 
on spin-space in any dimension), and $X$. denotes Clifford multiplication by $X$. Such a map always exists if $\mathfrak{S}$ is obtained by pulling-back to $\mathscr{S}$ a space-time spinor bundle, provided one has an externally oriented isometric embedding of $(\mathscr{S}, g)$ in a Lorentzian space-time available. Then the Clifford product $N \cdot$, where $N$ is the field of Lorentzian unit normals to the image of $\mathscr{S}$, has the required properties. Regardless of whether or not such a map exists, one can always replace $\mathfrak{S}$ by a direct sum of two copies of $\mathfrak{S}$; then, for $X \in T \mathscr{S}$, we let $X$. denote the Clifford action of $X$ and we set

$$
\begin{aligned}
& \gamma^{0}\left(\psi_{1}, \psi_{2}\right):=\left(\psi_{2}, \psi_{1}\right), \\
& X \cdot\left(\psi_{1}, \psi_{2}\right):=\left(X \cdot \psi_{1},-X \cdot \psi_{2}\right), \\
& D_{X}\left(\psi_{1}, \psi_{2}\right):=\left(D_{X} \psi_{1}, D_{X} \psi_{2}\right) .
\end{aligned}
$$

One checks that $(2.11 b)$ defines a representation of the Clifford algebra of $(\mathscr{S}, b)$ on $\mathfrak{S} \oplus \mathfrak{S}$, and that (4.6) holds.

One use of $\gamma^{0}$ is to construct Killing vectors for the metric $b$ out of imaginary Killing spinors. Indeed, if $\psi$ is such a spinor, and $e_{i}$ is a (locally defined) ON basis of $T \mathscr{S}$, then the vector field

$$
Y=\left\langle\psi, \gamma^{0} e^{i} \cdot \psi\right\rangle e_{i}
$$

is a Killing vector of the metric $b$. Furthermore, the pair $(V, Y)$, where $V=$ $\langle\psi, \psi\rangle$, defines a $b$-KID, by which we mean a $\operatorname{KID}$ of $(\mathscr{S}, b, 0)$.

We can now give our first result:

Theorem 2.1 (Positive charges theorem) Consider an initial data set $(\mathscr{S}, g, K)$ satisfying the positivity and fall-off conditions (2.5) and (2.1), with $(\mathscr{S}, \mathrm{g}) \mathrm{com}$ plete, and with finite total matter energy: $\rho \in L^{1}(\mathscr{S})$. We assume that either $\mathscr{S}$ has no boundary, or $\partial \mathscr{S}$ is compact and either (2.8) (changing $K$ to $-K$ if necessary) or (2.9) holds. Suppose that the Riemannian background metric $b$ admits imaginary Killing spinors in the asymptotic region, with respect to a spin structure which extends to the interior of $\mathscr{S}$. Let $\mathscr{K}_{0}$ be the subset of the set of b-KIDs which are of the form $\left(\langle\psi, \psi\rangle,\left\langle\psi, \gamma^{0} e^{i} \cdot \psi\right\rangle e_{i}\right)$ for some b-imaginary Killing spinor $\psi$. Then for all $X=(V, Y) \in \mathscr{K}_{0}$ we have

$$
H(V, Y) \geq 0
$$

with equality if and only if $\psi$ asymptotes to an imaginary Killing spinor of $(\mathscr{S}, g, K)$ associated with $\nabla$.

REMARK 2.2 It should be emphasised that the imaginary Killing spinors provided by Theorem 2.1 are only defined along $\mathscr{S}$, and not in an associated space-time if there is one.

REMARK 2.3 The bundle of spinors which is used in the proof is arbitrary. We will freely make use of this fact in our analysis in subsequent sections.

Proof: We use a Witten-type argument, as follows. Let $(\mathfrak{S},\langle\cdot, \cdot\rangle)$ be any Riemannian bundle of spinors over $(\mathscr{S}, g)$ with Hermitian product $\langle\cdot, \cdot\rangle$, such 
that Clifford multiplication (which we denote by ".") is anti-Hermitian, and with a map $\gamma^{0}$ satisfying (2.10).

Given an initial data set $(\mathscr{S}, g, K)$, a vector field $X$, and a spinor field $\xi$ we set

$$
\begin{aligned}
K(X) & :=K_{i}^{j} X^{i} e_{j} \cdot \\
\nabla_{X} \xi & :=D_{X} \xi+\frac{1}{2} K(X) \gamma^{0} \xi .
\end{aligned}
$$

Here $e_{i}$ is a local orthonormal basis of $T \mathscr{S}$; it is straightforward to check that (2.12) does not depend upon the choice of this basis.

The argument now has two main steps. First, one shows existence of a spinor $\chi$ satisfying a modified Dirac equation,

$$
e^{j} \cdot\left(\nabla_{j}+i \sqrt{\frac{-\Lambda}{2 n(n-1)}} e_{j} \cdot\right) \chi=0,
$$

and which asymptotes to $\psi$, where $\psi$ is an imaginary Killing spinor of the background metric. This can be done by rather obvious modifications of the arguments in [14], compare [35], see also [6,26] for the treatment of the boundary terms arising from a non-empty $\partial \mathscr{S}$. Let us simply point out that one of the ingredients of the proof is a weighted Poincaré inequality, established e.g. in [6] for the metrics of interest. This proves positivity of the boundary integral in the Witten identity. The next step is to prove that this boundary integral coincides with the Hamiltonian $H(V, Y)$. This is done by following the calculations in [3] and [14]. We note that the relevant part of those calculations does not use the explicit form of the imaginary Killing spinors, but only the equation satisfied by them.

\section{Spherical conformal infinity}

A preferred set of background Killing vector fields is provided by those which are $b$-normal to the initial data surface. The resulting Hamiltonians are usually interpreted as energies. In contradistinction with the asymptotically flat case, where only one normal background Killing vector field exists, if one assumes that conformal infinity has spherical space-like sections, then there are several normal background Killing vector fields. This implies that there is not a single energy, but rather an energy functional $M$. This functional $M$ is uniquely characterised by $n+1$ numbers $m_{(\mu)}, \mu=0,1, \ldots, n$, which transform as a Lorentz covector under asymptotic isometries ${ }^{5}$ of $g$, see [16,41]. (The component $m_{(0)}$ coincides with the Abbott-Deser mass under appropriate restrictions [16].) It follows that the Lorentzian length of $m_{(\mu)}$ is a geometric invariant of $(\mathscr{S}, g)$.

We start by reviewing the known $3+1$ results. The asymptotically-adSpositive-energy theorem implies that $m_{(\mu)}$ is causal, future pointing $[21,22,35]$

\footnotetext{
${ }^{5}$ These isometries are, essentially, characterised by conformal isometries of the conformal boundary at infinity (in the current case the sphere).
} 
(compare $[14,41,42])$. If it vanishes, then $(\mathscr{S}, g, K)$ are initial data for anti-de Sitter space-time. ${ }^{6}$

Quite generally, one can view the hyperbolic space as a unit spacelike hyperboloid in $\mathbb{R}^{n+1}$, the latter equipped with the Minkowski metric. If one assumes that $m_{(\mu)}$ is timelike, after applying an asymptotic isometry to obtain $m_{(\mu)}=(m, 0, \cdots, 0)$, the background Killing vector fields tangent to $\mathscr{S}$ can now be split into rotations and "boosts". In space-time dimension four it is customary to define the rest-frame angular momentum as

$$
j_{(i)}:=H\left(0, \beta_{(i)}\right) \text {, }
$$

where the $\beta_{(i)}$ 's are the generators of rotations of $S^{2}$, when embedded in $\mathbb{R}^{3}$ :

$$
\beta_{(i)}=\epsilon_{i j k} x^{j} \partial_{k}
$$

The numerical values of the remaining three Hamiltonians, associated with the vector fields $C_{(i)}$ of (3.6) below, generating boost transformations, will be denoted by $c_{(i)}$. In the asymptotically flat case the $c_{(i)}$ 's have the interpretation of the centre of mass of the system, and can always be set to zero by a translation of the coordinates. This freedom does not exist in the asymptotically adS situation. We will retain the name centre of mass for the vector $\vec{c}=\left(c_{(i)}\right)$.

It does not appear to be widely known that, in 3+1-dimensions, the positive energy theorem for asymptotically adS initial data implies an upper bound on the center of mass and the angular momentum in terms of $m$. This should be contrasted with the asymptotically Minkowskian positive energy theorem, which bounds the space-momentum in terms of the energy, but does not impose constraints either on angular momentum or on centre of mass. ${ }^{7}$ Recall that with our choices so far the energy-momentum vector $m_{(\mu)}$ lies along the time axis. A rotation of the coordinate system aligns the angular momentum vector $\vec{j}$ along the first coordinate axis. One can then rotate $\vec{c}=\left(c_{(i)}\right)$ to lie in the $x-y$ plane. It is shown in [35] that the positivity theorem 2.1 implies the following inequality

$$
m \geq \sqrt{-\Lambda / 3} \sqrt{\left(\left|j_{(1)}\right|+\left|c_{(2)}\right|\right)^{2}+c_{(1)}^{2}},
$$

with vanishing $m$ if and only if the initial data set arises from anti-de Sitter space-time. $^{8}$

\footnotetext{
${ }^{6}$ In fact, the proof of this in [35] contains a gap which we fill, see the proof of Theorem 3.9, end of Section 3.1 below.

${ }^{7}$ Schoen (seminar at the ESI, summer 2003) has shown that there is no bound on the ratio $|\vec{j}| / m$ for vacuum initial data sets with $\Lambda=0$.

${ }^{8}$ The normalisations of the Hamiltonians are a matter of conventions, ours are as follows: the mass $m_{(0)}$ is the numerical value of the Hamiltonian associated with the background Killing vector $\partial_{t}$ when the background adS metric is written in the form $-\left(1-\Lambda r^{2} / n(n-\right.$ 1)) $d t^{2}+\left(1-\Lambda r^{2} / n(n-1)\right)^{-1} d r^{2}+r^{2} d \Omega^{2}$, where $d \Omega^{2}$ is the unit round metric on the $(n-1)$ dimensional sphere. This normalisation is convenient for comparison with the $\Lambda=0$ limit. Next, the angular momentum is the numerical value of the Hamiltonian associated with the rotations of $S^{n-1}$ normalised so that a rotation by $2 \pi$ is the identity. Finally, the center of mass is normalised to make the right-hand-side of our inequalities look simple.
} 
The inequality (3.1) can be rewritten in the manifestly rotation-invariant form

$$
m \geq \sqrt{-\Lambda / 3} \sqrt{|\vec{c}|^{2}+|\vec{j}|^{2}+2|\vec{c} \times \vec{j}|},
$$

where $\vec{c} \times \vec{j}$ is the vector product, while $|\vec{j}|=\sqrt{j_{(1)}^{2}+j_{(2)}^{2}+j_{(3)}^{2}}$, etc. In particular we have the striking upper bounds

$$
m \geq \sqrt{-\Lambda / 3}|\vec{j}|, \quad m \geq \sqrt{-\Lambda / 3}|\vec{c}|
$$

Thus, both the length of the angular momentum vector and that of the centre of mass vector are bounded by (a multiple of) the invariant norm of the mass functional $M$.

The first inequality in (3.3) is a familiar condition in the explicit family of Kerr-adS metrics (see, e.g., [25]). Thus, the restriction on the range of parameters stemming from the Kerr-adS family is not a result of our incomplete knowledge of the set of all solutions, but a necessary property of non-singular asymptotically adS space-times satisfying the dominant energy condition.

The above leaves several questions unanswered and suggest others: is there an equivalent of $(3.2)$ when $m_{(\mu)}$ is null? what happens if the inequalities are equalities? what if ${ }^{n-1} M$ is a two-dimensional torus? what happens in higher dimensions? In this work we give partial or complete answers to these questions.

First some notation: from now on, in space-time dimension $n$, we view the hyperbolic space as the open unit ball $B^{n}(1) \subset \mathbb{R}^{n}$ equipped with the metric $b={ }^{n} b=\omega^{-2} \delta$, where $\delta$ is the standard flat metric on $\mathbb{R}^{n}$, and

$$
\omega=\frac{1-|x|^{2}}{2} .
$$

In the obvious spin frame associated with this conformal representation ${ }^{9}$, the imaginary Killing spinors of ${ }^{n} b$ take the form

$$
\psi_{u}=\omega^{-1 / 2}\left(1-i x^{k} \gamma^{k}\right) u
$$

(summation over $k$ ), where $u$ is a spinor with constant entries, while the antiHermitian matrices $\gamma^{k}$ with constant entries satisfy the flat space Clifford relations

$$
\gamma^{i} \gamma^{j}+\gamma^{j} \gamma^{i}=-2 \delta^{i j}
$$

(The $\psi_{u}$ 's exhaust the space of imaginary Killing spinors because the map which assigns $u$ to $\psi_{u}(0)$ is a bijection). As already mentioned, we will also need a Hermitian matrix $\gamma^{0}$, with constant entries, satisfying

$$
\left(\gamma^{0}\right)^{2}=1, \quad \gamma^{0} \gamma^{j}+\gamma^{j} \gamma^{0}=0 .
$$

(If such a matrix does not exist we first make a doubling construction on the $u$ 's as in (2.11).) The KID $\left(V_{u}, Y_{u}^{i}\right)$ associated to $\psi_{u}$ takes the form

$$
V_{u}:=\left\langle\psi_{u}, \psi_{u}\right\rangle=2(|u|^{2} \underbrace{\frac{1+|x|^{2}}{1-|x|^{2}}}_{=: V_{(0)}}+\left\langle u, i \gamma^{k} u\right\rangle \underbrace{\frac{(-2) x^{k}}{1-|x|^{2}}}_{=: V_{(k)}}),
$$

\footnotetext{
${ }^{9}$ More precisely, we take a spin frame which projects to the frame $\theta^{i}=\omega^{-1} d x^{i}$, and a local basis of the spinor bundle in which the $\gamma^{\mu}$ s are constant matrices.
} 


$$
\begin{aligned}
Y_{u}^{i} \partial_{i} & :=\left\langle\psi_{u}, \gamma^{0} \gamma^{i} \psi_{u}\right\rangle e_{i} \\
& =2\left\langle u, \gamma^{0} \gamma^{k} u\right\rangle \underbrace{\left(\frac{1+|x|^{2}}{2} \delta_{k}^{i}-x^{i} x^{k}\right) \partial_{i}}_{:=C_{(k)}}+\frac{1}{2}\left\langle u, i \gamma^{0}\left(\gamma^{k} \gamma^{i}-\gamma^{i} \gamma^{k}\right) u\right\rangle \underbrace{\left(x_{k} \partial_{i}-x_{i} \partial_{k}\right)}_{:=\Omega_{(k)(i)}}
\end{aligned}
$$

The KIDs $\left(V_{(\mu)}, 0\right), \mu=0, \ldots, n$, together with $\left(0, C_{(k)}\right), k=1, \ldots n$, and $\left(0, \Omega_{(i)(j)}\right), 1 \leq i<j \leq n$, span the space of KIDs of $(B(1), b, 0)$. The $\Omega_{(i)(j)}$ 's obviously generate rotations, and therefore it is natural to use the name angular momenta for the corresponding global charges; these will be denoted by $J_{(i)(j)}$. As shown in $[16,41]$, the collection of functions $\left(V_{(0)}, V_{(1)}, \ldots, V_{(n)}\right)$, transforms as a Lorentz covector under conformal isometries of the boundary at infinity. This is at the origin of the name energy-momentum vector, denoted by $m_{(\mu)}$, for the associated charges. As already mentioned at the beginning of this section, the $C_{(k)}$ 's generate Lorentz boosts, when the hyperbolic space is embedded as a hyperboloid into $(n+1)$-dimensional Minkowski space; the associated charges will be denoted by $c_{(k)}$, and called center of mass.

It will be convenient to reduce $J_{(k)(j)}$ to a canonical form. As a matrix $J_{(k)(j)}$ is anti-symmetric, so that there exists an ON-frame in which $J_{(k)(j)}$ is block-diagonal, built out of two-by-two blocks of the form

$$
\left[\begin{array}{cc}
0 & \omega_{(i)} \\
-\omega_{(i)} & 0
\end{array}\right]
$$

with furthermore a last column of zeros in odd space-dimension.

Our next result is the following:

TheOREM 3.1 Let $\mathscr{S}$ be spin with $\operatorname{dim} \mathscr{S}=n \geq 3$ and suppose that ${ }^{n-1} M=$ $S^{n-1}$ (then the spin structures on $\mathscr{S}$ and $\mathscr{S}_{\text {ext }}$ are necessarily compatible). Under the remaining hypotheses of Theorem 2.1, $m_{(\mu)}$ is causal future ${ }^{10}$ directed, or vanishes. Furthermore,

1. In every conformal frame $e^{11}$ it holds that

$$
\ell m_{(0)} \geq\left|\omega_{(1)}\right|+\left|\omega_{(2)}\right|+\ldots+\left|\omega_{(l)}\right|
$$

where

$$
\ell:=\sqrt{-\frac{n(n-1)}{2 \Lambda}}
$$

2. If $m_{(\mu)}$ is null, then the space of $\nabla$-imaginary Killing sections of $\mathfrak{S} \oplus \mathfrak{S}$ over $\mathscr{S}$ (as defined in (2.11)) is at least $\operatorname{dim} \mathfrak{S}$-dimensional.

\footnotetext{
${ }^{10}$ The notion of causality of $m_{(\mu)}$ is determined by a Lorentzian metric with signature $(1, n)$ defined by the group of isometries of hyperbolic space [16], with "future" defined as $m_{(0)}>0$.

${ }^{11}$ Recall that the decomposition of $g$ as a background plus a correction term involves a choice, and that two such choices can be related to each other by a conformal transformation of the conformal boundary at infinity, plus higher order corrections [16]. We use the term "conformal frame" to emphasise the fact that such a choice has been made.
} 
3. When $m_{(\mu)}$ is timelike we also have, in a frame where $m_{(i)}=0$,

$$
\ell m_{(0)} \geq \sqrt{c_{(1)}^{2}+\cdots+c_{(n)}^{2}} .
$$

4. If $m_{(0)}$ vanishes in some conformal frame, then all global charges vanish, and the space-time metric along $\mathscr{S}$ is Einstein with vanishing Weyl tensor.

5. In dimension $5+1$, in a specific frame which will be defined in the proof below, we have the stronger inequality, which is optimal:

$$
\ell m \geq \sqrt{c_{(1)}^{2}+c_{(3)}^{2}+c_{(5)}^{2}+\omega_{(1)}^{2}+\omega_{(2)}^{2}+2 \sqrt{\left(\omega_{(1)} c_{(1)}\right)^{2}+\left(\omega_{(2)} c_{(3)}\right)^{2}+\left(\omega_{(1)} \omega_{(2)}\right)^{2}}} .
$$

6. Inequality (3.11) remains valid and optimal in dimension $4+1$ after setting $c_{(5)}=0$.

7. Similarly (3.11) remains valid and optimal in dimension $3+1$ after setting $c_{(5)}=\omega_{(2)}=0$, and is then identical to (3.2).

REMARK 3.2 Equation (3.11) suggests that in all dimensions the following (nonoptimal) inequality should hold

$$
\ell m_{(0)} \geq \sqrt{\sum_{i} c_{(i)}^{2}+\left(\sum_{i<j}\left|J_{(i)(j)}\right|\right)^{2}} .
$$

REMARK 3.3 A class of $4+1$ dimensional examples with $m_{(0)} \neq 0$ saturating the bound (3.8) is given by the metrics in [24] with $F_{\mu \nu}^{I}=0$, or the metrics in [19].

REMARK 3.4 We will see in Section 3.1 below that, in dimension $3+1$, under natural hypotheses $m_{(\mu)}$ cannot be null.

Proof: To avoid annoying multiplicative factors involving the dimension and the cosmological constant, all calculations that follow are done assuming $\Lambda=$ $-n(n-1) / 2$, so that the background hyperbolic metric has all sectional curvatures equal to one. This can be achieved by a scaling of the metric; the general result is then obtained by rescaling back.

We have

$$
\begin{aligned}
& H\left(V_{u}, Y_{u}^{i}\right)= 2 H\left(|u|^{2}\left(V_{(0)}, 0\right)+\left\langle u, i \gamma^{k} u\right\rangle\left(V_{(k)}, 0\right)\right. \\
&\left.+\left\langle u, \gamma^{0} \gamma^{k} u\right\rangle\left(0, C_{(k)}\right)+\frac{1}{4}\left\langle u, i \gamma^{0}(\underbrace{\gamma^{k} \gamma^{j}-\gamma^{j} \gamma^{k}}_{=: 2 \gamma^{k j}}) u\right\rangle\left(0, \Omega_{(k)(j)}\right)\right) \\
&=2(|u|^{2} \underbrace{H\left(V_{(0)}, 0\right)}_{m_{(0)}}+\left\langle u, i \gamma^{k} u\right\rangle \underbrace{H\left(V_{(k)}, 0\right)}_{m_{(k)}} \\
&+\left\langle u, \gamma^{0} \gamma^{k} u\right\rangle \underbrace{H\left(0, C_{(k)}\right)}_{c_{(k)}}+\frac{1}{2}\left\langle u, i \gamma^{0} \gamma^{k j} u\right\rangle \underbrace{H\left(0, \Omega_{(k)(j)}\right)}_{J_{(k)(j)}})
\end{aligned}
$$




$$
=2\langle u, \underbrace{\left(m_{(0)}+i \gamma^{k} m_{(k)}+\gamma^{0} \gamma^{k} c_{(k)}+\frac{1}{2} i \gamma^{0} \gamma^{k j} J_{(k)(j)}\right)}_{=: Q} u\rangle .
$$

By the positivity theorem 2.1 the matrix $Q$ must be positive semi-definite. Let us explore the consequences thereof.

We start by restricting our considerations to spinors $u$ satisfying

$$
\gamma^{0} u= \pm u
$$

and $|u|^{2}=1$ (recall that $\gamma^{0}$ is Hermitian, and its eigenvalues are plus or minus one since its square is one). As $\gamma^{i}$ anti-commutes with $\gamma^{0}$, it maps $( \pm 1)-$ eigenspinors of $\gamma^{0}$ to $(\mp 1)$-eigenspinors; thus $\gamma^{i} u$ and $\gamma^{0} \gamma^{i} u$ are each orthogonal to $u$. We conclude that, on the eigenspaces of $\gamma^{0}$, the following holds

$$
\langle u, Q u\rangle=\left\langle u,\left(m_{(0)}+\frac{i}{2} \gamma^{0} \gamma^{k j} J_{(k)(j)}\right) u\right\rangle .
$$

For $n=3$ (compare (3.7)) we have

$$
\frac{1}{2} i \gamma^{0} \gamma^{k j} J_{(k)(j)}=\omega_{(1)} i \gamma^{0} \gamma^{1} \gamma^{2}
$$

while in higher dimensions $2 l \leq n \leq 2 l+1$ we can write

$$
\frac{1}{2} i \gamma^{0} \gamma^{k j} J_{(k)(j)}=\omega_{(1)} i \gamma^{0} \gamma^{1} \gamma^{2}+\omega_{(2)} i \gamma^{0} \gamma^{3} \gamma^{4}+\ldots+\omega_{(l)} i \gamma^{0} \gamma^{2 l-1} \gamma^{2 l}
$$

The matrices $i \gamma^{0} \gamma^{2 k-1} \gamma^{2 k}$ are Hermitian, with square one, therefore their eigenvalues are plus or minus one. We will need the following:

LEMMA 3.5 For every collection $\left\{\epsilon_{a}\right\}_{a=0, \ldots, l}$, with $\epsilon_{a}^{2}=1$, after performing a doubling of $\mathfrak{S}$ if necessary as in (2.11), there exists $u$ satisfying $\gamma^{0} u=\epsilon_{0} u$ and

$$
\forall a \geq 1 \quad i \gamma^{0} \gamma^{2 a-1} \gamma^{2 a} u=\epsilon_{a} u .
$$

REMARK 3.6 The result is wrong without the doubling in general, which can be seen by taking $n=2, \gamma^{1}=i \sigma^{1}, \gamma^{2}=i \sigma^{2}$, and $\gamma^{0}=\sigma^{3}$, where the $\sigma^{i}$ 's are the usual two-by-two Pauli matrices.

Proof: The matrix $i \gamma^{2 l-1} \gamma^{2 l}$ is Hermitian, with square one, therefore its eigenvalues are plus or minus one. The matrix $\gamma^{2 l}$ defines a bijection between the $(+1)$-eigenspace and the $(-1)$-eigenspace, so that each of these spaces is nonempty. Let $X_{l}$ denote the $\epsilon_{0} \epsilon_{l}$-eigenspace of $i \gamma^{2 l-1} \gamma^{2 l}$. For $0 \leq \mu \leq 2 l-2$ the matrices $\gamma^{\mu}$ commute with $i \gamma^{2 l-1} \gamma^{2 l}$, which implies that $X_{l}$ is invariant under their action. For $l \geq 3$ we repeat this construction to obtain a subspace $X_{l-1} \subset X_{l}$ on which $i \gamma^{2 l-3} \gamma^{2 l-2}=\epsilon_{0} \epsilon_{l-1}$. After $l$ steps we obtain a space $X_{0} \subset X_{1} \subset \ldots \subset X_{l}$ which is invariant under $\gamma^{0}$. If there exists a spinor $u$ in $X_{0}$ such that $\gamma^{0} u=\epsilon_{0} u$, the result immediately follows. Otherwise we double $\mathfrak{S}$ as in (2.11), we take $\hat{u}$ to be any non-zero element of $X_{0}$, and we set $u=\left(\hat{u}, \epsilon_{0} \hat{u}\right)$. 
Let $u$ be given by Lemma 3.5 with $\epsilon_{a}=-\operatorname{sgn} \omega_{(a)}$. We obtain

$$
0 \leq\langle u, Q u\rangle=\left(m_{(0)}-\left|\omega_{(1)}\right|-\ldots-\left|\omega_{(l)}\right|\right)|u|^{2},
$$

proving point 1:

$$
m_{(0)} \geq\left|\omega_{(1)}\right|+\ldots+\left|\omega_{(l)}\right| .
$$

In particular $m_{(0)}$ is non-negative. Since conformal transformations of the sphere at infinity induce Lorentz transformations of $m_{(\mu)}$ we obtain that $m_{(\mu)}$ is causal future directed, or vanishes. Equality implies that the boundary integral in the Witten identity vanishes, and the volume integral shows that $u$ is an imaginary Killing spinor (on $\mathscr{S}$ ) for the modified connection (2.13).

If $m_{(\mu)}$ is timelike we clearly also have

$$
m \geq\left|\omega_{(1)}\right|+\ldots+\left|\omega_{(l)}\right|,
$$

where

$$
m:=\sqrt{\left|\eta^{(\mu)(\nu)} m_{(\mu)} m_{(\nu)}\right|},
$$

with $\eta^{(\mu)(\nu)}=\operatorname{diag}(-1,+1, \ldots,+1)$, and the $\omega_{(i)}$ 's in (3.15) are the angular momenta in a Lorentz frame in which $m_{(\mu)}$ is aligned along the time axis.

Still assuming timelikeness of $M:=\left(m_{(\mu)}\right)$, and choosing an ON frame in which $M$ is aligned along $e_{(0)}$, we now drop the condition (3.12) and assume that $n=3$. We retain (3.13), and make a rotation in the $\left\{e_{1}, e_{2}\right\}$ plane so that $c_{(2)}=0$. Since the Hermitian matrices $\gamma^{0} \gamma^{1}$ and $i \gamma^{0} \gamma^{1} \gamma^{2}$ commute, and square to one, we can choose $u_{1}$ such that $\left|u_{1}\right|^{2}=1$ and, replacing $\gamma^{1}$ by $-\gamma^{1}$ and $\gamma^{2}$ by $-\gamma^{2}$ if necessary,

$$
i \gamma^{0} \gamma^{1} \gamma^{2} u_{1}=u_{1}, \quad \gamma^{0} \gamma^{1} u_{1}=u_{1}
$$

Set

$$
u_{2}:=\gamma^{0} \gamma^{3} u_{1}, \quad u_{3}:=\gamma^{0} \gamma^{2} u_{1}, \quad u_{4}:=\gamma^{0} \gamma^{3} u_{3}=-\gamma^{3} \gamma^{2} u_{1} .
$$

From the Clifford relations one easily finds that

$$
\left(\begin{array}{l}
Q u_{1} \\
Q u_{2} \\
Q u_{3} \\
Q u_{4}
\end{array}\right)=\left(\begin{array}{cccc}
m+\left(c_{(1)}+\omega_{(1)}\right) & c_{(3)} & 0 & 0 \\
c_{(3)} & m-\left(c_{(1)}+\omega_{(1)}\right) & 0 & 0 \\
0 & 0 & m+\left(-c_{(1)}+\omega_{(1)}\right) & c_{(3)} \\
0 & 0 & c_{(3)} & m-\left(-c_{(1)}+\omega_{(1)}\right)
\end{array}\right)\left(\begin{array}{l}
u_{1} \\
u_{2} \\
u_{3} \\
u_{4}
\end{array}\right)
$$

One can further check that the $u_{i}$ 's form an ON basis as follows: $u_{1}$ is orthogonal to $u_{2}$ because both are eigenvectors of the Hermitian matrix $i \gamma^{0} \gamma^{1} \gamma^{2}$ with different eigenvalues. (This can be verified by inspecting the sign in front of $\omega_{(1)}$ in the matrix above.) For the same reason $u_{1}$ is orthogonal to $u_{4}$, and $u_{2}$ is orthogonal to $u_{3}$. It remains to justify orthogonality of the elements of the pair $\left(u_{1}, u_{3}\right)$, similarly for $\left(u_{2}, u_{4}\right)$. These follow from the fact that the first spinor in each of those pairs is an eigenvector of $\gamma^{0} \gamma^{1}$ with an eigenvalue different from the second one in the pair. ${ }^{12}$

\footnotetext{
${ }^{12}$ If one uses a space of spinors which carries an irreducible representation of the Clifford algebra, than the above matrix describes $Q$ completely. Otherwise one can, using descending induction, find an $\mathrm{ON}$ basis in which $Q$ is block-diagonal, with blocks as above.
} 
Thus, the $u_{i}$ 's form an ON basis of Vect $\left\{u_{i}\right\}$, so that the positivity properties of $Q$, when restricted to this subspace, can be read off by calculating the eigenvalues of the matrix above. These are easily found to be

$$
m \pm \sqrt{\left(c_{(1)} \pm \omega_{(1)}\right)^{2}+c_{(3)}^{2}} .
$$

In particular we have rederived the property that $Q$ is non-negative if and only if Maerten's inequality (3.2) holds. Furthermore, there will be at least two linearly independent imaginary Killing spinors if and only if the kernel of $Q$ is at least two-dimensional. Under the current hypotheses, and assuming an irreducible representation of the Clifford algebra, this will happen if and only if

$$
c_{(1)} \omega_{(1)}=0 \quad \Longleftrightarrow J_{(i)(j)} c^{j}=0 \quad \Longleftrightarrow \vec{j} \times \vec{c}=0 .
$$

We now return to general dimension, also dropping the assumption that $m_{(\mu)}$ is timelike. We use spinors obtained by the "doubling" technique as in (2.11); it then follows that the matrix $Q$ has the following block structure:

$$
Q=\left(\begin{array}{cc}
m_{(0)}+i \gamma^{k} m_{(k)} & \underbrace{-\gamma^{k} c_{(k)}+\frac{i}{2} J_{(k)(l)} \gamma^{k} \gamma^{l}}_{=: B} \\
\gamma^{k} c_{(k)}+\frac{i}{2} J_{(k)(l)} \gamma^{k} \gamma^{l} & m_{(0)}-i \gamma^{k} m_{(k)}
\end{array}\right)
$$

(Positivity of $Q$ when restricted to spinors of the form $(u, 0)$ gives immediately that $m_{(\mu)}$ is causal future pointing, which we already know.)

Suppose that $m_{(\mu)}$ is null, then there exists a $\left(\frac{1}{2} \operatorname{dim} \mathfrak{S}\right)$-dimensional space of $u \in \mathfrak{S}$ such that $\left(m_{(0)}+i \gamma^{k} m_{(k)}\right) u=0$. Likewise there exists a $\left(\frac{1}{2} \operatorname{dim} \mathfrak{S}\right)-$ dimensional space of $v \in \mathfrak{S}$ such that $\left(m_{(0)}-i \gamma^{k} m_{(k)}\right) v=0$. Applying $Q$ to a pair $(u, \lambda v)$, where $\lambda \in \mathbb{C}$, with such an $u$ and $v$, we obtain

$$
0 \leq\langle(u, \lambda v), Q(u, \lambda v)\rangle=2 \Re(\langle u, \lambda B v\rangle) .
$$

Since $\lambda$ is arbitrary we conclude that $\langle(u, v), Q(u, v)\rangle=0$. Thus, the space of pairs $(u, v)$ which lead to a zero Hamiltonian charge $H$ equals at least $\frac{1}{2} \operatorname{dim} \mathfrak{S}+\frac{1}{2} \operatorname{dim} \mathfrak{S}=\operatorname{dim} \mathfrak{S}$. Witten's identity shows that each such $u$ leads to an imaginary $\nabla$-Killing spinor of $(\mathscr{S}, g, K)$, section of $\mathfrak{S} \oplus \mathfrak{S}$. This proves point 2 .

Suppose, next, that $m_{(\mu)}$ is timelike, and let us use a conformal frame in which $m_{(k)}=0$. Using a spinor of the form $(u, i u)$ one obtains instead

$$
0 \leq\langle(u, i u), Q(u, i u)\rangle=2 \Re\left(\left\langle u,\left(m_{(0)}+i B\right) u\right\rangle\right)=2\left\langle u,\left(m_{(0)}-i \gamma^{k} c_{(k)}\right) u\right\rangle
$$

for all $u$, proving (3.10).

To prove point 4 , suppose that $m_{(0)}$ vanishes, then $m_{(k)}=0$ by causality of $m_{(\mu)}$, further $J_{(k)(\ell)}$ vanishes by (3.8). Applying (3.18) to spinors of the form $(u, \pm v)$, positivity of $Q$ implies $c_{(k)}=0$. Thus $Q$ vanishes, which implies that the space of Killing spinors has maximal dimension. One concludes that the space-time metric is Einstein, with vanishing Weyl tensor, along $\mathscr{S}$ by the 
calculations in [35, Section 4], which are done there for $n=3$, but remain valid for larger values of $n$.

In order to establish our remaining claims, we describe now an attempt to obtain a simple form of $Q$ in higher dimensions. While part of the calculation that follows can be done in any dimension, we have only been able to carry it out completely in dimensions $4+1$ and $5+1$. We assume that $m_{(k)}$ is timelike, and we use an ON frame adapted to $m_{(k)}$ in which (3.14) holds. In each plane Vect $\left\{e_{2 j-1}, e_{2 j}\right\}$ we further make a rotation so that $c_{(2 j)}=0$. Let $l$ be such that $2 l \leq n \leq 2 l+1$, for $1 \leq j \leq l$ set

$$
B_{j}:=i \gamma^{0} \gamma^{2 j-1} \gamma^{2 j}, \quad A_{j}=\gamma^{0} \gamma^{2 j-1}
$$

then the $A_{i}$ 's and $B_{i}$ 's are Hermitian, with square one, and satisfy the commutation relations

$$
B_{i} B_{j}=B_{j} B_{i}, \quad B_{i} A_{j}=\left\{\begin{array}{ll}
-A_{j} B_{i}, & i \neq j ; \\
A_{j} B_{i}, & i=j,
\end{array} \quad A_{i} A_{j}= \begin{cases}-A_{j} A_{i}, & i \neq j \\
A_{j} A_{i}, & i=j\end{cases}\right.
$$

Changing some of the $\gamma^{k}$ 's to $-\gamma^{k}$ 's if necessary, we can find a spinor $u$ such that

$$
\forall i \quad B_{i} u=u .
$$

Setting

$$
u_{i}:=A_{i} u,
$$

one easily obtains the $B_{j} u_{i}$ 's using (3.19):

$$
B_{j} u_{i}= \begin{cases}-u_{i}, & i \neq j \\ u_{i}, & i=j\end{cases}
$$

For $n=6$ we can enlarge Vect $\left\{u_{0}:=u, u_{1}, u_{2}, u_{3}\right\}$ to a space which is invariant under the action of the $A_{i}$ 's by adding, to the generating family $\left\{u_{\mu}\right\}$, the spinors $u_{4}:=A_{1} A_{2} u, u_{5}:=A_{1} A_{3} u, u_{6}:=A_{2} A_{3} u$, and $u_{7}:=A_{1} A_{2} A_{3} u$. It is then easy to work out the matrix of $Q$ in that basis (by considerations similar to the ones after (3.16) one checks that the $u_{\mu}$ 's form an orthonormal family); we only report the result for $\omega_{(3)}=0$; for typesetting reasons we write $b_{i}$ for $\omega_{(i)}$ and $a_{i}$ for $c_{(2 i-1)}$ :

$\left[\begin{array}{cccccccc}m+b_{1}+b_{2} & a_{1} & a_{2} & a_{3} & 0 & 0 & 0 & 0 \\ a_{1} & m+b_{1}-b_{2} & 0 & 0 & -a_{2} & -a_{3} & 0 & 0 \\ a_{2} & 0 & m-b_{1}+b_{2} & 0 & a_{1} & 0 & -a_{3} & 0 \\ a_{3} & 0 & 0 & m-b_{1}-b_{2} & 0 & a_{1} & a_{2} & 0 \\ 0 & -a_{2} & a_{1} & 0 & m-b_{1}-b_{2} & 0 & 0 & a_{3} \\ 0 & -a_{3} & 0 & a_{1} & 0 & m-b_{1}+b_{2} & 0 & -a_{2} \\ 0 & 0 & -a_{3} & a_{2} & 0 & 0 & m+b_{1}-b_{2} & a_{1} \\ 0 & 0 & 0 & 0 & a_{3} & -a_{2} & a_{1} & m+b_{1}+b_{2}\end{array}\right]$


One can use Maple or Mathematica to compute the eigenvalues of $Q$ without assuming $\omega_{(3)}=0$, but this does not lead to useful expressions. However, suppose that $n=5$; after embedding the five-dimensional Clifford algebra into a six dimensional one, this form of a general $Q$ holds in the basis above. A MAPLE calculation shows then that the eigenvalues of $Q$ on this subspace all have multiplicity two, and are equal to

$m \pm \sqrt{c_{(1)}^{2}+c_{(3)}^{2}+c_{(5)}^{2}+\omega_{(1)}^{2}+\omega_{(2)}^{2} \pm 2 \sqrt{\left(\omega_{(1)} c_{(1)}\right)^{2}+\left(\omega_{(2)} c_{(3)}\right)^{2}+\left(\omega_{(1)} \omega_{(2)}\right)^{2}}}$.

This gives (3.11).

Specialising further to $c_{(5)}=0$, a similar argument gives the inequality for $n=4$, proving point 6 ; a further specialisation leads to point 7 .

\subsection{Impossibility of null energy-momentum when $n=3$}

Under the hypotheses of Theorem 2.1, equality in (3.2) leads to the existence of imaginary $\nabla$-Killing spinors on $\mathscr{S}$. We have the following result, which does not assume a spherical conformal boundary:

TheOREM 3.7 Let $\operatorname{dim} \mathscr{S}=3$, and suppose that $(\mathscr{S}, g, K)$ admits a non-trivial imaginary Killing spinor for the connection (2.13). Then:

1. The Killing development of $(\mathscr{S}, g, K)$ admits an imaginary Killing spinor.

2. If there are two linearly independent such spinors on $\mathscr{S}$, then the Killing development of $(\mathscr{S}, g, K)$ is vacuum and has vanishing Weyl tensor.

REMARK 3.8 In higher dimensions, the minimal number of Killing spinors which enforces the vanishing of the Weyl tensor does not appear to be known. For example, consider a five-dimensional Lorentzian Einstein-Sasaki manifold (all regular types can be constructed as $S^{1}$-bundles over Kähler-Einstein manifolds with negative scalar curvature, see $[8,11,31])$. A Lorentzian Einstein-Sasaki space is not conformally flat and has (if it is simply connected) at least two linearly independent imaginary Killing spinors. ${ }^{13}$ In those examples we can choose $\operatorname{dim} \mathfrak{S}=4$, leading to dimension four of the space of doubled imaginary Killing spinors in point 2 of Theorem 3.1. Restricting to an irreducible subrepresentation of the Clifford algebra will presumably lead to a two-dimensional space, so that our constraints on a null $m_{(\mu)}$ do not exclude such non-trivial geometries. In fact, five-dimensional examples with a two-dimensional space of imaginary Killing spinors can be found within the family described in Section 4.4, with a toroidal Scri; but note that these do not have a null $m_{(\mu)}$.

Proof of Theorem 3.7: For point 1, we need to show that existence of space imaginary Killing spinors for (2.13), that is spinors satisfying the following:

$$
\widehat{\nabla}_{X} \psi \equiv D_{X} \psi+\frac{1}{2} K(X) \cdot \gamma^{0} \psi+i \sqrt{\frac{-\Lambda}{2 n(n-1)}} X \cdot \psi=0, \quad X \in T \mathscr{S},
$$

\footnotetext{
${ }^{13}$ We are grateful to Helga Baum for those remarks.
} 
necessarily implies that of space-time imaginary Killing spinors in the Killing development of $(\mathscr{S}, g, K)$.

We will prove the result using Dirac spinors on $\mathscr{S}$, and especially their decomposition into two component spinors, which simplifies the calculations. We use Greek indices for space-time, preserving Latin indices for some of the lower dimensional situations which follow; two-component spinor indices will be capital Latin indices as usual (see [37] for further two-spinor conventions; note, however, the opposite signature of the metric here).

A space-time imaginary Killing spinor $\psi$ can then be represented by a pair of spinor fields $\left(\alpha_{A}, \beta_{A^{\prime}}\right)$ satisfying the following coupled system of equations (compare [36, Section 2]):

$$
\begin{aligned}
\nabla_{A A^{\prime}} \alpha_{B} & =b \epsilon_{A B} \beta_{A^{\prime}}, \\
\nabla_{A A^{\prime}} \beta_{B^{\prime}} & =b \epsilon_{A^{\prime} B^{\prime}} \alpha_{A},
\end{aligned}
$$

where $b$ is a constant (not to be confused with the background metric of Section 2), which without loss of generality may be assumed real, and is then related to the cosmological constant by $\Lambda=-6 b^{2}$.

Saturation of (3.2) implies that the data $(\mathscr{S}, g, K)$ admits a spinor field $\psi$ satisfying the projection into $\mathscr{S}$ of $(3.21)$, say

$$
\Pi_{\gamma}^{\alpha} S_{\alpha}=0
$$

where $\Pi_{\gamma}^{\alpha}$ is the tensor projecting tangentially to $\mathscr{S}$ and $S_{\alpha}$ stands for:

$$
S_{\alpha}:=\left(\begin{array}{c}
\nabla_{A A^{\prime}} \alpha_{B}-b \epsilon_{A B} \beta_{A^{\prime}} \\
\nabla_{A A^{\prime}} \beta_{B^{\prime}}-b \epsilon_{A^{\prime} B^{\prime}} \alpha_{A}
\end{array}\right) .
$$

Given a solution $\left(\alpha_{A}, \beta_{A^{\prime}}\right)$ of $(3.21)$, another solution is provided by $\left(\bar{\beta}_{A}, \bar{\alpha}_{A^{\prime}}\right)$. The two solutions are linearly independent unless $\alpha_{A}$ and $\bar{\beta}_{A}$ are proportional, say $\alpha_{A}=f \bar{\beta}_{A}$ for some function $f$. In this case, it follows from (3.21) that $f$ is a complex constant, of modulus one, and it can then be absorbed into a redefinition of $\beta_{A^{\prime}}$. Thus, given a solution of (3.21), we necessarily have at least a two-dimensional space of solutions unless we have a solution of

$$
\nabla_{A A^{\prime} O_{B}}=b \epsilon_{A B} \bar{o}_{A^{\prime}}
$$

For reasons which will appear, we shall call this the null case, while a solution of (3.21) not of this form we call the non-null case.

Assuming that the full (as opposed to (3.22)) system (3.21) holds, by commuting derivatives one finds

$$
\begin{aligned}
& \psi_{A B C D} \alpha^{D}=0=\bar{\psi}_{A^{\prime} B^{\prime} C^{\prime} D^{\prime}} \beta^{D^{\prime}} \\
& \phi_{A B A^{\prime} B^{\prime}} \alpha^{B}=0=\phi_{A B A^{\prime} B^{\prime}} \beta^{B^{\prime}}
\end{aligned}
$$

where $\psi_{A B C D}$ is the Weyl spinor, the spinor representing the Weyl tensor, and $\phi_{A B A^{\prime} B^{\prime}}$ is the Ricci spinor, representing the trace-free part of the Ricci tensor. In the non-null case, when $\alpha_{A}$ and $\bar{\beta}_{A}$ are linearly independent, it follows from 
this that the Weyl and trace-free Ricci tensors both vanish and the space-time is locally anti-de Sitter.

For non-trivial examples, therefore, we need to be in the null case. From (3.24) by differentiating again we obtain

$$
\psi_{A B C D} O^{D}=0=\phi_{A B A^{\prime} B^{\prime}} O^{B},
$$

so that

$$
\psi_{A B C D}=\Psi o_{A} o_{B} o_{C} o_{D}, \quad \phi_{A B A^{\prime} B^{\prime}}=\Phi o_{A} o_{B} \bar{o}_{A^{\prime}} \bar{o}_{B^{\prime}},
$$

for complex functions $\Psi$ and $\Phi$.

Even in the null case, if there are two linearly-independent such solutions, we shall again have, locally, anti-de Sitter space (since $\psi_{A B C D}$ and $\phi_{A B A^{\prime} B^{\prime}}$ cannot take this form for two independent spinors).

We return now to (3.22). Suppose first that we are in the non-null case. The vector $X^{\alpha}$ constructed according to

$$
X^{\alpha}=\alpha^{A} \bar{\alpha}^{A^{\prime}}+\bar{\beta}^{A} \beta^{A^{\prime}},
$$

will give [35] 'Killing Initial Data' at $\mathscr{S}$. In the Killing development of $(\mathscr{S}, g, K)$, $X^{\alpha}$ will be a future-pointing, timelike Killing vector. From (3.25) we see, at $\mathscr{S}$

$$
X^{\alpha} X_{\alpha}=-2 V \bar{V}
$$

where

$$
V=\alpha_{A} \bar{\beta}^{A}
$$

By (3.22) we have

$$
\Pi_{\mu}^{\alpha} \nabla_{\alpha} V=\Pi_{\mu}^{\alpha} b\left(\alpha_{A} \bar{\alpha}_{A^{\prime}}-\bar{\beta}_{A} \beta_{A^{\prime}}\right),
$$

which is real so that the imaginary part of $V$, say $I$, is necessarily a constant along $\mathscr{S}$.

Recall that the Lie-derivative of a spinor field $\alpha_{A}$ along a Killing vector $L^{\alpha}$ is defined as

$$
\mathscr{L}_{L} \alpha_{A}:=L^{\mu} \nabla_{\mu} \alpha_{A}+\Phi_{A}^{M} \alpha_{M}
$$

where the symmetric spinor $\Phi_{M N}$ is defined by

$$
\nabla_{\mu} L_{\nu}=\Phi_{M N} \epsilon_{M^{\prime} N^{\prime}}+\bar{\Phi}_{M^{\prime} N^{\prime}} \epsilon_{M N},
$$

see e.g. [29, p. 40]. For $X^{\alpha}$, from (3.25) and (3.22) we find, at $\mathscr{S}$,

$$
\Pi_{\alpha}^{\mu} \nabla_{\mu} X_{\beta}=2 b \Pi_{\alpha}^{\mu}\left(\alpha_{(M} \bar{\beta}_{B)} \epsilon_{M^{\prime} B^{\prime}}+\bar{\alpha}_{\left(M^{\prime}\right.} \beta_{\left.B^{\prime}\right)} \epsilon_{M B}\right),
$$

so that, at $\mathscr{S}$ in the Killing development,

$$
\nabla_{\alpha} X_{\beta}=2 b\left(\alpha_{(A} \bar{\beta}_{B)} \epsilon_{A^{\prime} B^{\prime}}+\bar{\alpha}_{\left(A^{\prime}\right.} \beta_{\left.B^{\prime}\right)} \epsilon_{A B}\right)+n_{\alpha} v_{\beta},
$$

for some vector field $v_{\beta}$ where $n_{\alpha}$ is the (unit, timelike) normal to $\mathscr{S}$. Symmetrising over the indices $\alpha$ and $\beta$ the left-hand-side vanishes, thus so does the right-hand-side, which implies $v_{\alpha}=0$. Thus the derivative at $\mathscr{S}$ of $X$ is

$$
\nabla_{\alpha} X_{\beta}=2 b\left(\alpha_{(A} \bar{\beta}_{B)} \epsilon_{A^{\prime} B^{\prime}}+\bar{\alpha}_{\left(A^{\prime}\right.} \beta_{\left.B^{\prime}\right)} \epsilon_{A B}\right),
$$


so that

$$
\Phi_{A B}=2 b \alpha_{(A} \bar{\beta}_{B)}
$$

We impose

$$
\mathscr{L}_{X} \alpha_{A}-2 i b I \alpha_{A}=0=\mathscr{L}_{X} \beta_{A^{\prime}}-2 i b I \beta_{A^{\prime}}
$$

in the Killing development, with $\alpha_{A}$ and $\beta_{A^{\prime}}$ known on $\mathscr{S}$ and $I$ the value of the (constant) imaginary part of $V$ at $\mathscr{S}$. This determines the spinors throughout the Killing development. Note also that now $\mathscr{L}_{X} V=0$ in the Killing development, so that $I$ equals $\Im V$ throughout. Furthermore, it follows that

$$
\mathscr{L}_{X}\left(\alpha_{(A} \bar{\beta}_{B)}\right)=0
$$

so that the Lie derivative along $X$ of both sides of (3.30) vanishes, and therefore this equation holds throughout the Killing development.

From (3.31), (3.30) and (3.28), we now have

$$
X^{\alpha} S_{\alpha}=0
$$

with $S_{\alpha}$ as in (3.23), and from (3.31)

$$
\mathscr{L}_{X} S_{\alpha}=2 i b I S_{\alpha}
$$

Since $X^{\alpha}$ is transversal to $\mathscr{S}$, this with (3.22) gives $S_{\alpha}=0$ at $\mathscr{S}$, and therefore throughout the Killing development. Now we have a solution of (3.21) in the Killing development, which is therefore locally anti-de Sitter.

The null case is very similar: now we have a solution of

$$
\Pi_{\gamma}^{\alpha} S_{\alpha}=0
$$

where this time $S_{\alpha}$ stands for

$$
S_{\alpha}:=\nabla_{A A^{\prime}} O_{B}-b \epsilon_{A B} \bar{o}_{B^{\prime}}
$$

We define the Killing vector by

$$
X^{\alpha}=o^{A} \bar{o}^{A^{\prime}} .
$$

This is a future-pointing null vector (which is why we called this the null case). Since $V$ is now zero, (3.31) becomes

$$
\mathscr{L}_{X} O_{A}=0
$$

and we proceed as before.

For the derivative of $X$ we find in this case

$$
\nabla_{\alpha} X_{\beta}=b o_{A} o_{B} \epsilon_{A^{\prime} B^{\prime}}+b \bar{o}_{A^{\prime}} \bar{o}_{B^{\prime}} \epsilon_{A B} .
$$

It follows that

$$
X^{\alpha} S_{\alpha}=0
$$

at $\mathscr{S}$, so that again $S_{\alpha}$ vanishes at $\mathscr{S}$, but now $\mathscr{L}_{X} S_{\alpha}=0$. We conclude as required that $S_{\alpha}$ vanishes in the Killing development.

We shall say that $(\mathscr{S}, g, K)$ are smooth at infinity if the corresponding initial data for the conformally rescaled metric are smooth at the conformal boundary at infinity. We have the following corollary of Theorem 3.7: 
TheOREm 3.9 Under the hypotheses of Theorem 2.1 let $\operatorname{dim} \mathscr{S}=3$, assume that the conformal boundary at infinity $\dot{\mathscr{S}}$ is a finite collection of spheres, with the metric satisfying the decay conditions (2.1) in each of the asymptotic regions. Suppose moreover that the conformally completed manifold $\mathscr{S} \cup \dot{\mathscr{S}}$ is compact. If $(\mathscr{S}, g, K)$ is smooth at infinity then:

1. If $(\mathscr{S}, g, K)$ admits two linearly independent imaginary Killing spinors for the connection (2.13) (which will be true if $m_{(0)}$ vanishes), then the initial data set arises from a hypersurface in anti-de Sitter space-time.

2. $m_{(\mu)}$ cannot be null.

3. Equality in (3.2) together with $\vec{j} \times \vec{c}=0$ (equivalently, $\omega_{(1)} c_{(1)}=0$ ) occurs if and only if $(\mathscr{S}, g, K)$ can be obtained from a hypersurface in anti-de Sitter space-time.

REMARK 3.10 The condition that $(\mathscr{S}, g, K)$ is smooth at infinity ensures equality of the Witten boundary integral with Ashtekar's formula for mass in terms of the Weyl tensor, and can be weakened by working out the differentiability threshold needed for this equality.

Proof of Theorem 3.9: 1. By Theorem 3.7, or by point 4 of Theorem 3.1 if $m_{(0)}$ vanishes, the Weyl and Ricci tensors vanish so that the Killing development is locally anti-de Sitter. To prove that it is globally anti-de Sitter, it suffices (compare the arguments in [35, Theorem 1.4]) to prove that it is geodesically complete $^{14}$. This will be a consequence of the following Lemma, provided that we can show that its hypotheses are satisfied:

LEMMA 3.11 In space-time dimension $n+1 \geq 2$, consider a stationary Lorentzian metric

$$
{ }^{n+1} g=-\exp (\mu)(d t+\underbrace{\theta_{i} d x^{i}}_{=: \theta})^{2}+h,
$$

on $\mathscr{M}:=\mathbb{R}_{t} \times \mathscr{S}$, where $h$ is a complete Riemannian metric on $\mathscr{S}$, with Killing vector $X=\partial_{t}$ satisfying

$$
\exp (\mu):=-g(X, X) \geq \varepsilon, \quad|\theta|_{h} \leq \varepsilon^{-1}
$$

for some constant $\varepsilon>0$. Then $\left(\mathscr{M},{ }^{n+1} g\right)$ is geodesically complete.

REMARK 3.12 This lemma together with the remaining arguments of the proof below shows that, in all dimensions $n \geq 3$, the vanishing of $m_{(0)}$ implies that the data set arises from the anti-de Sitter space-time whenever the subspace of KIDs generated by those arising from spinors contains a KID satisfying (3.38). We will show that this is necessarily true when $n=3$; to generalise our result to all dimensions one would need to justify (3.38) for $n \geq 4$.

\footnotetext{
${ }^{14}$ The proof of geodesic completeness of the Killing development of $(\mathscr{S}, g, K)$ in [35, Theorem 1.4] invokes [1, Lemma 1.1]. However, that last lemma is incorrect. A counter-example is given by the domain of outer communications of an extreme Reissner-Nordström black hole.
} 
Proof: Let $\Gamma(s)=(t(s), \lambda(s))$ be an affinely parameterised maximally extended geodesic in $\left(\mathscr{M},{ }^{n+1} g\right)$, set

$$
\epsilon:={ }^{n+1} g(\dot{\Gamma}, \dot{\Gamma}), \quad p:={ }^{n+1} g(\dot{\Gamma}, X)=-e^{\mu}(\dot{t}+\theta(\dot{\lambda})),
$$

thus $\epsilon$ and $p$ are constant along $\Gamma$. Hence

$$
h(\dot{\lambda}, \dot{\lambda})=\epsilon+e^{-\mu} p^{2} \leq C
$$

for some constant $C$, and then

$$
|\dot{t}|=\left|e^{-\mu} p+\theta(\dot{\lambda})\right| \leq C^{\prime}
$$

for some other constant $C^{\prime}$. This implies that for any bounded interval $I \subset \mathbb{R}$ the closure $\overline{\Gamma(I)}$ of the image $\Gamma(I) \subset \mathscr{M}$ is compact, and completeness of $\left(\mathscr{M},{ }^{n+1} g\right)$ readily follows.

Returning to the proof of Theorem 3.9, since the Weyl tensor vanishes, it follows e.g. from [27] that in each of the asymptotic regions the Witten boundary term is identically zero. ${ }^{15}$ Hence the matrix $Q$ vanishes, and there is an imaginary Killing spinor $\chi_{u}$ at $\mathscr{S}$ for every choice of imaginary $b$-Killing spinor $\psi_{u}$ in each asymptotic end, with $\chi_{u}$ asymptoting to zero in all the remaining ends. However, the number of imaginary Killing spinors is at most equal to the number of imaginary $b$-Killing spinors in one end; it follows that $\mathscr{S}$ can only have one asymptotic region.

We show that at least one of these imaginary Killing spinors leads to a Killing vector which is timelike everywhere on $\mathscr{S}$, with controlled $\mu$ and $\theta$.

By (3.21), for any space-time Killing spinor, we have

$$
\nabla_{\alpha}\left(\alpha_{A} \bar{\beta}^{A}-\bar{\alpha}_{A^{\prime}} \beta^{A^{\prime}}\right)=0
$$

so that the imaginary part $\Im V$ of $V=\alpha_{A} \bar{\beta}^{A}$ is constant. By choosing the Killing spinor so that this is not zero at some point $p$ on $\mathscr{S}$ we ensure that it is nonzero everywhere. But the Killing vector $X$ of (3.25) has norm

$$
-{ }^{4} g(X, X)=2 V \bar{V} \geq 2(\Im V)^{2}>0,
$$

so that the Killing vector is everywhere timelike.

By Lemma 3.5 there exist spinors $u_{ \pm, 0}$ such that, in any dimension $n \geq 3$, for $a=1, \ldots,\lfloor n / 2\rfloor$,

$$
\left|u_{ \pm, 0}\right|=1, \quad \gamma^{0} u_{ \pm, 0}=u_{ \pm, 0}, \quad i \gamma^{0} \gamma^{2 a-1} \gamma^{2 a} u_{ \pm, 0}= \pm u_{ \pm, 0}
$$

The calculations in (3.5)-(3.6) show that the resulting $b$-KIDs lead, respectively, to the following $b$-Killing vectors

$$
\partial_{t} \pm\left(\sum_{a=1}^{l} \Omega_{(2 a-1)(2 a)}\right) .
$$

\footnotetext{
${ }^{15}$ In [27] the existence of a space-time with a global $\mathscr{I}$ is assumed, but the calculations there can be repeated in our context.
} 
Adding, we conclude that the vector subspace of $b$-KIDs generated by imaginary $b$-Killing spinors contains the vector $\partial_{t}$.

Recall, next, that under the current conditions $(\mathscr{S}, g, K)$ has the maximal number of imaginary Killing spinors. This implies that for every $p \in \mathscr{S}$ the map which to $u$ assigns the value $\chi_{u}(p)$, where $\chi_{u}$ is the solution of the Witten equation which asymptotes to $\psi_{u}$, is a linear bijection. Now, the equation $\Im V(p)=0$ defines an algebraic variety in the space of spinors at $p$, the complement of which is open and dense. Therefore there exists an open and dense set of $u$ 's such that the corresponding $\chi_{u}$ 's will have $\Im V(p) \neq 0$, and consequently will lead to timelike KIDs. (A KID is called timelike if the associated Killing vector is.)

Set

$$
r=\frac{1}{1-|x|}
$$

where $x$ is a coordinate as in (3.4). It follows from (3.5)-(3.6), together with the asymptotics of solutions of the Witten equation, that for any $\epsilon>0$ we can choose $u_{ \pm, \epsilon}$ so that $u_{ \pm, \epsilon}$ approaches $u_{ \pm, 0}$ as $\epsilon$ tends to zero, and the corresponding KIDs $\left(V_{ \pm, \epsilon}, Y_{ \pm, \epsilon}\right)$ are timelike, with, for large $r$,

$$
V_{\epsilon}:=V_{+, \epsilon}+V_{-, \epsilon}=(1+O(\epsilon)) r, \quad|\underbrace{Y_{+, \epsilon}+Y_{-, \epsilon}}_{=: Y_{\epsilon}}|_{g}=O(\epsilon) r .
$$

(Note that a sum of timelike future oriented KIDs is timelike.)

We consider the Killing development defined by $\left(V_{\epsilon}, Y_{\epsilon}\right)$, with $\epsilon$ sufficiently small so that $V_{\epsilon} \geq 2 r / 3$ and $\left|Y_{\epsilon}\right|_{g} \leq r / 3$ : thus $\mathscr{M}$ is $\mathbb{R}_{t} \times \mathscr{S}$ with metric

$$
{ }^{4} g=-V_{\epsilon}^{2} d t^{2}+g_{i j}\left(d x^{i}+Y_{\epsilon}^{i} d t\right)\left(d x^{j}+Y_{\epsilon}^{j} d t\right),
$$

with Killing vector $X=\partial_{t}$. Letting

$$
\exp (\mu):=-{ }^{4} g(X, X)=V_{\epsilon}^{2}-\left|Y_{\epsilon}\right|_{g}^{2} \geq \frac{r^{2}}{3}
$$

we rewrite the space-time metric ${ }^{4} g$ as in (3.37),

$$
{ }^{4} g=-\exp (\mu)\left(d t+\theta_{i} d x^{i}\right)^{2}+h,
$$

so that

$$
\theta_{i}=-e^{-\mu} g_{i j} Y_{\epsilon}^{j} .
$$

The asymptotics (2.1) of $g$, together with compactness of $\mathscr{S} \cup \dot{\mathscr{S}}$ and the Hopf-Rinow theorem imply completeness of $(\mathscr{S}, g)$. The Riemannian metric $h$ is related to the initial data metric $g$ by the equation

$$
h_{i j}=g_{i j}+\exp (\mu) \theta_{i} \theta_{j}
$$

and, since the last term gives a non-negative contribution on any given vector, completeness of $(\mathscr{S}, h)$ follows from that of $(\mathscr{S}, g)$.

The function $\mu$ is uniformly bounded away from zero by (3.39)-(3.40) and, for all $\epsilon$ sufficiently small, it tends to infinity as one recedes to infinity on $\mathscr{S}_{\text {ext }}$ by $(3.41)$. 
Finally, from (3.44) and (3.41), $e^{\mu / 2}|\theta|_{g}$ is uniformly bounded in (each of) the asymptotic regions, and the norm with respect to $h$ is equivalent to that with respect to $g$ (with error terms of order $\epsilon^{2}$ ). Since $\mathscr{S}$ is a union of a compact set and one asymptotic end where $\theta$ has already been shown to be controlled, a uniform bound on $|\theta|_{h}$ follows. Point 1 follows now from Lemma 3.11.

2: By point 2 of Theorem 3.1 there are at least two linearly independent Killing spinors, and the result follows from point 1 of the current theorem.

3: As pointed out in the paragraph preceding (3.17) the hypotheses of point 1 are satisfied.

\subsection{Non-existence of black hole solutions saturating the equal- ity, $n=3$}

Whatever the dimension $n \geq 3$, there exist higher-genus Kottler black hole space-times with zero Hamiltonian mass. One could naively think of those as saturating our positivity bounds. However, it should be borne in mind that, for reasons already explained, those solutions (as well as any solutions with the same asymptotic behavior) do not possess imaginary Killing spinors, so our inequality does not apply.

We wish to show, under a natural supplementary assumption, non-existence of $(3+1)$-dimensional black hole space-times (not necessarily vacuum), with spherical or toroidal conformal infinity, saturating the angular momentum bound, except perhaps for Weyl-flat solutions. To be precise, in addition to the hypotheses of the positivity theorem 2.1 , we will assume that $\mathscr{S}$ is the union of an asymptotically hyperbolic region $\mathscr{S}_{\text {ext }}$ and of a compact set, with non-empty smooth boundary. Moreover, we suppose that the space-time $\left(\mathscr{M},{ }^{4} g\right)$ is not conformally flat. ${ }^{16}$ The hypothesis that the bound is saturated implies existence of a Killing spinor, and thus also of the associated Killing vector which we call $X$, which must be null by the analysis of Section 3.1. The hypothesis of existence of a black hole will be encoded in the assumption that the boundary of $\mathscr{S}$, when moved by the flow of the Killing vector field $X$, forms ${ }^{17}$ a null hypersurface $\mathscr{H}$. So, assume for contradiction that a solution satisfying the above exists. Then $X$ is necessarily tangent to the generators of $\mathscr{H}$, with zero surface gravity since $X$ is null everywhere. It follows from (3.35) that the solution is static in the sense that $X^{b} \wedge d X^{b}=0$, where $X^{b}={ }^{4} g(X, \cdot)$. In vacuum this implies [17] that the horizon has higher genus topology. But this contradicts [20, Theorem 4.1], showing that vacuum solutions of the above kind are not possible. Finally, the reader will easily check that the hypothesis that the space-time is vacuum plays no role in this argument, because the energy-momentum tensor of the space-time metric is necessarily proportional to $X \otimes X$, and such a tensor does not affect those equations in [17] which are

\footnotetext{
${ }^{16}$ This hypothesis is used to prove that the horizon is degenerate. It can be replaced by the condition that the imaginary Killing spinor extends smoothly across the event horizon, as then the associated Killing vector will be causal, which again enforces degeneracy.

${ }^{17}$ Note that the level sets of $u$ are null hypersurfaces generated by $X$, but with non-compact intersection with $\mathscr{S}$.
} 
relevant to the problem at hand, so that there are no non-vacuum black holes satisfying these conditions either.

\subsection{Siklos waves}

Theorem 3.9 shows that for nontrivial examples saturating Maerten's inequality we need there to be just a one-dimensional family of solutions of (3.24). Metrics with this property will be briefly described in this section. We have seen in Section 3.2 that, subject to some natural restrictions, such examples do not include black hole solutions. In fact, we shall see in Section 3.4 that such examples are not possible at all in three space dimensions if we further assume that $\mathscr{I}$ has spherical cross-sections and is "large enough".

The "Lobatchevski plane waves" of Siklos [38], which we propose to call Siklos waves, are precisely characterised by the existence of a nontrivial spinor satisfying (3.24). Siklos shows that it is possible to introduce coordinates so that the metric may be written as

$$
g=\frac{1}{2 b^{2} x^{2}}\left(d x^{2}+d y^{2}-2 d u d v-H(u, x, y) d u^{2}\right) .
$$

Here $X=\partial / \partial v$. (The signature of (3.45) is reversed as compared to [38].) The Weyl and Ricci spinors are

$$
\begin{aligned}
\phi_{A B A^{\prime} B^{\prime}} & =\Phi o_{A} o_{B} \bar{o}_{A^{\prime}} \bar{o}_{B^{\prime}} \\
\psi_{A B C D} & =\Psi o_{A} o_{B} o_{C} o_{D}
\end{aligned}
$$

where $\Phi$ and $\Psi$ are given in terms of $H$ by ${ }^{18}$

$$
\begin{aligned}
& \Phi=-b^{4} x^{4}\left(H_{x x}+H_{y y}-2 H_{x} / x\right) . \\
& \Psi=-b^{4} x^{4}\left(H_{x x}-H_{y y}-2 i H_{x y}\right)
\end{aligned}
$$

The cosmological constant is $\Lambda=-6 b^{2}$ (this is not the $\Lambda$ of the Newman-Penrose formalism which would be $24 b^{2}$ ).

The Killing vector $X$ Lie drags the Weyl spinor (since it is a symmetry) and the Lie derivative defined by (3.28) commutes with contractions and tensor products, so that from $(3.47)$

$$
X^{\alpha} \partial_{\alpha} \Psi=0
$$

If $H$ is zero, then (3.45) is the metric of anti-de Sitter space with $\mathscr{I}$ at $x=0$. A variety of other choices for the function $H$ also lead to anti-de Sitter space, in particular a constant, say $H=H_{0}$, as the coordinate transformation

$$
d v \rightarrow d V=d v+\frac{1}{2} H_{0} d u
$$

demonstrates.

\footnotetext{
${ }^{18}$ The multiplicative factor $1 / 16$ in the equation for $\Phi_{22}$ in $[38$, p. 254] should be $1 / 4$.
} 


\subsection{Rigidity in the $n=3$ spherical case}

In Section 3.1 we have shown that a null $m_{(\mu)}$ cannot occur. In this section we wish to show that the remaining possibilities for equality in (3.2) only occur in anti-de Sitter space-time, under the supplementary condition ${ }^{19}$ that the initial data set arises from a space-time with a conformal completion at infinity which is "sufficiently large in time". By this we mean that the interval of the $t$ coordinate below has length at least $\pi$.

A (four-dimensional) space-time $\left(\mathscr{M},{ }^{4} g\right)$ is said to be asymptotically-antide Sitter if it is smoothly conformal to a manifold $\widetilde{\mathscr{M}}$ with boundary $\partial \widetilde{\mathscr{M}} \equiv$ $\mathscr{I} \approx \mathbb{R} \times S^{2}$, with the usual condition that the conformal factor $\Omega$, relating the metrics as ${ }^{4} g=\Omega^{-2}{ }^{4} \tilde{g}$, vanishes on $\mathscr{I}$ precisely at order one. It is further assumed that the restriction of ${ }^{4} \tilde{g}$ to the conformal boundary at infinity equals

$$
\stackrel{\circ}{h}_{i j} d x^{i} d x^{j}=d \theta^{2}+\sin ^{2} \theta d \phi^{2}-d t^{2} .
$$

It is then possible to introduce ${ }^{20}$ coordinates $\left(R, x^{i}\right)$ for $i=1,2,3$ so that the space-time metric ${ }^{4} g=g$ can be written in the form

$$
g=\frac{1}{R^{2}}\left(d R^{2}+h_{i j}\left(R, x^{k}\right) d x^{i} d x^{j}\right)
$$

with

$$
h_{i j}=\stackrel{\circ}{h}_{i j}\left(x^{k}\right)+O\left(R^{2}\right) .
$$

The metric (3.45) with $2 b^{2}=1$ and $H=0$ takes this form, though the metric of anti-de Sitter space is more commonly written as

$$
g=d \psi^{2}+\sinh ^{2} \psi\left(d \theta^{2}+\sin ^{2} \theta d \phi^{2}\right)-\cosh ^{2} \psi d t^{2},
$$

when the substitution $R=\mathrm{e}^{-\psi}$ will cast it in the form of (3.52).

Our aim now is to show that the metric of the Siklos wave (3.45) cannot be written in the asymptotically-anti-de Sitter form (3.52) unless it is exactly anti-de Sitter. Our technique will be, first to obtain an asymptotic form of the Killing vector $X$ and then to show that the equation (3.50) is incompatible with (3.52) unless $\Psi=0$.

We suppose then that $X$ is a Killing vector for the metric (3.52) and write it in the form

$$
X=A \frac{\partial}{\partial R}+B^{i} \frac{\partial}{\partial x^{i}}
$$

The Killing equation may be written as

$$
X^{\gamma} \partial_{\gamma} g_{\alpha \beta}+g_{\alpha \gamma} \partial_{\beta} X^{\gamma}+g_{\gamma \beta} \partial_{\alpha} X^{\gamma}=0 .
$$

Substituting from (3.52) we obtain for the (00) component of this

$$
R \frac{\partial A}{\partial R}-A=0
$$

\footnotetext{
${ }^{19}$ This hypothesis can often be removed by using the Killing development. This is, unfortunately, not the case for the problem at hand because of the zeros of the Killing vector at $\mathscr{I}$.

${ }^{20}$ In this section, and only in this section, we use the convention that $x^{0}=R$; the reader should not confuse this with a time-coordinate.
} 
so that

$$
A=R V\left(x^{i}\right)
$$

for some $V\left(x^{i}\right)$, to be found. For the $(0 i)$ components we find

$$
\frac{\partial A}{\partial x^{i}}+h_{i j} \frac{\partial B^{j}}{\partial R}=0
$$

so that

$$
B^{j}\left(R, x^{k}\right)=B_{0}^{j}\left(x^{k}\right)+O\left(R^{2}\right) .
$$

Finally, for the $(i j)$ components we find

$$
\mathscr{L}_{B} h_{i j}=2 V h_{i j}-R V \frac{\partial}{\partial R} h_{i j}
$$

The leading term in this equation, with what we have already, requires

$$
\mathscr{L}_{B_{0}} \stackrel{\circ}{h}_{i j}=2 V \stackrel{\circ}{h}_{i j}
$$

Thus $B_{0}$ is a conformal Killing vector on $\mathscr{I}$, and our next task is to find these. We proceed as before, by setting

$$
B_{0}^{i} \frac{\partial}{\partial x^{i}}=\beta \frac{\partial}{\partial t}+A^{a} \frac{\partial}{\partial y^{a}}
$$

where $a=2,3$ and $\left(y^{2}, y^{3}\right)=(\theta, \phi)$. This is to be a conformal Killing vector of the metric (3.51) which we write as

$$
\stackrel{\circ}{h}_{i j} d x^{i} d x^{j}=\eta_{a b} d y^{a} d y^{b}-d t^{2} .
$$

The conformal Killing equation (3.58) may be written in the form

$$
B_{0}^{k} \partial_{k} \stackrel{\circ}{h}_{i j}+\stackrel{\circ}{h}_{i k} \partial_{j} B_{0}^{k}+\stackrel{\circ}{h}_{k j} \partial_{i} B_{0}^{k}=2 V \stackrel{\circ}{h}_{i j}
$$

from which, as before, we obtain the system of equations

$$
\begin{aligned}
\frac{\partial \beta}{\partial t} & =V \\
\frac{\partial \beta}{\partial y^{a}} & =\eta_{a b} \frac{\partial A^{b}}{\partial t} \\
\mathscr{L}_{A} \eta_{a b} & =2 V \eta_{a b} .
\end{aligned}
$$

To solve these, we need to know some facts about conformal Killing vectors on $S^{2}$ (which, by (3.62), $A=A^{a} \partial_{a}$ is). The general solution of (3.62) is of the form:

$$
A^{a}=Z^{a}-\eta^{a b} \frac{\partial \alpha}{\partial y^{b}}
$$

where $Z^{a}$ is a Killing vector for $\eta$ and $\alpha$ is a conformal scalar, by which we mean a solution of the equation:

$$
\mathscr{D}_{a} \mathscr{D}_{b} \alpha=-\alpha \eta_{a b}
$$


where $\mathscr{D}_{a}$ is the Levi-Civita covariant derivative for $\eta$. Thus, from (3.62), $V=\alpha$. Next, it now follows from (3.61) that

$$
\frac{\partial}{\partial y^{a}}\left(\beta+\frac{\partial \alpha}{\partial t}\right)=\eta_{a b} \frac{\partial Z^{b}}{\partial t} .
$$

Taking the divergence of this we find that

$$
\frac{\partial Z^{a}}{\partial t}=0 ; \quad \beta=\beta_{0}-\frac{\partial \alpha}{\partial t}
$$

for some $\beta_{0}$ independent of $y^{a}$. Finally, integrating (3.60) over $\mathbf{S}^{2}$, and noting that $\alpha$ integrates to zero because of the equation $\Delta \alpha=-2 \alpha$, shows that $\beta_{0}$ is actually constant and $\alpha$ satisfies

$$
\frac{\partial^{2} \alpha}{\partial t^{2}}=-\alpha
$$

which is readily solved.

We may write out solutions explicitly by regarding the $\mathbf{S}^{2}$ as the unit sphere in $\mathbb{R}^{3}$ with Cartesian coordinates $\mathbf{X}=\left(X^{\mathbf{i}}\right), \mathbf{i}=1,2,3$. Then $\alpha$ is linear in $X^{\mathbf{i}}$ and, taking account of (3.66), may be written in the form

$$
\alpha=-(\mathbf{a} \cdot \mathbf{X}) \cos t-(\mathbf{b} \cdot \mathbf{X}) \sin t
$$

in terms of a pair of constant vectors $\mathbf{a}$ and $\mathbf{b}$. By (3.65) we obtain

$$
\beta=\beta_{0}-(\mathbf{a} \cdot \mathbf{X}) \sin t+(\mathbf{b} \cdot \mathbf{X}) \cos t,
$$

while $Z$ is a Killing vector, so that

$$
Z^{a} \frac{\partial}{\partial y^{a}}=M_{\mathbf{i j}} X^{\mathbf{i}} \frac{\partial}{\partial X^{\mathbf{j}}}
$$

for a constant, antisymmetric matrix $M_{\mathbf{i j}}$ (where necessary, indices $\mathbf{i}, \mathbf{j}$ can be raised or lowered with $\delta_{\mathbf{i j}}$ ).

We have found an asymptotic form for the most general Killing vector of (3.52). However, we are interested in null Killing vectors, so that by (3.55)

$$
g(X, X):=\frac{1}{R^{2}}\left(A^{2}+h_{i j} B^{i} B^{j}\right)=0,
$$

which implies in particular that

$$
\stackrel{\circ}{h}_{i j} B_{0}^{i} B_{0}^{j}=0
$$

so that $B_{0}$ is also null.

From (3.59) and (3.63) this is the condition

$$
-\beta^{2}+\eta^{a b}\left(Z_{a}-\partial_{a} \alpha\right)\left(Z_{b}-\partial_{b} \alpha\right)=0 .
$$

Substituting into this from (3.67), (3.68) and (3.69), we obtain a series of algebraic equations by equating to zero coefficients of $1, \sin t, \cos t$ and $\cos 2 t$. These are

$$
|\mathbf{a}|^{2}=|\mathbf{b}|^{2} ; \quad \mathbf{a} \cdot \mathbf{b}=0
$$


then

$$
\begin{aligned}
\beta_{0} a_{\mathbf{i}}+M_{\mathbf{i j}} b_{\mathbf{j}} & =0 \\
-\beta_{0} b_{\mathbf{i}}+M_{\mathbf{i j}} a_{\mathbf{j}} & =0
\end{aligned}
$$

so that

$$
M_{\mathbf{i j}}=\epsilon\left(a_{\mathbf{i}} b_{\mathbf{j}}-a_{\mathbf{j}} b_{\mathbf{i}}\right)
$$

for constant $\epsilon$, and finally

$$
-\beta_{0}^{2}+M_{\mathbf{i k}} M_{\mathbf{j} k} X^{\mathbf{i}} X^{\mathbf{j}}+|\mathbf{a}|^{2}-(\mathbf{a} \cdot \mathbf{X})^{2}-(\mathbf{b} \cdot \mathbf{X})^{2}=0,
$$

which implies just

$$
\beta_{0}=-\epsilon|\mathbf{a}|^{2}
$$

with $\epsilon^{2}|\mathbf{a}|^{2}=1$.

We have found the general form of any null Killing vector in any asymptotically adS space-time, so that $X$ of Section 3.3 must have this form, in any Siklos wave which is asymptotically adS. There are two families depending on the sign of $\epsilon$ and the six real parameters (a, b) subject to (3.71). Replacing the Killing spinor by a multiple thereof if necessary, we can without loss of generality assume $|\mathbf{a}|=1$. All choices are equivalent up to rotation and the discrete symmetry $t \rightarrow t+\pi / 2$. We make the choices

$$
a_{1}=b_{2}=\epsilon=1
$$

with other terms zero, then with $\mathbf{X}=(\sin \theta \cos \phi, \sin \theta \sin \phi, \cos \theta)$ we obtain

$$
\alpha=-\sin \theta \cos (\phi-t)
$$

and

$$
\begin{aligned}
B_{0}= & (-1+\sin \theta \sin (\phi-t)) \frac{\partial}{\partial t}+\cos \theta \cos (\phi-t) \frac{\partial}{\partial \theta} \\
& +(1-\sin (\phi-t) \csc \theta) \frac{\partial}{\partial \phi} .
\end{aligned}
$$

Now we have the Killing vector $X$, at least asymptotically, we need to solve (3.50). For this we need the asymptotic form of $\Psi$ compatible with (3.52). We recall some of the conventions associated with conformal rescaling in asymptotically adS space-times. The unphysical metric is

$$
\tilde{g}_{\alpha \beta}=R^{2} g_{\alpha \beta}
$$

with $R$ and $g_{\alpha \beta}$ as in (3.52). From (3.55) and (3.56), we see that the Killing vector $X$ extends to a smooth vector field on $\mathscr{I}$, and we have

$$
\nabla_{\mu} X_{\nu}=\frac{2}{R^{3}} \tilde{X}_{[\mu} \partial_{\nu]} R+O\left(R^{-2}\right)
$$

where $\tilde{X}_{\mu}:=\tilde{g}_{\mu \nu} X^{\nu}$; here and below $O\left(R^{k}\right)$ refers to components in the coordinate system $\left(R, x^{i}\right)$. From (3.30), where now $b=1 / \sqrt{2}$, and (3.47), the Weyl tensor $W$ equals

$$
W=A(d X \otimes d X-(* d X) \otimes(* d X))+B(d X \otimes(* d X)+(* d X) \otimes d X),
$$


where $*$ is the space-time Hodge-dual, while

$$
\Psi=A-i B
$$

and $\Psi$ is as in (3.47) and (3.49). Let $N=R \partial_{R}$ be a unit normal to the level sets of $R$.

Recall that the electric and magnetic Weyl tensors at a hypersurface with normal $N$ are defined as $E_{i j}:=W_{i \gamma j \delta} N^{\gamma} N^{\delta}$ and $B_{i j}:=* W_{i \gamma j \delta} N^{\gamma} N^{\delta}$ respectively, where $W$ is the Weyl tensor and $* W$ is its dual. The rescaled electric Weyl tensor, finite on $\mathscr{I}$ (see [27, Lemma 3.1] ) is, by equation (2.14) of [4] and by $(3.76)-(3.77)$

$$
\begin{aligned}
\tilde{E}_{i j} & =R^{-1} W_{i \gamma j \delta} N^{\gamma} N^{\delta} \\
& =R^{-5}\left(A \tilde{X}_{i} \tilde{X}_{j}+O(\Psi R)\right) .
\end{aligned}
$$

Similarly the rescaled magnetic part of the Weyl tensor, finite on $\mathscr{I}$, is

$$
\begin{aligned}
\tilde{B}_{i j} & =R^{-1} * W_{i \gamma j \delta} N^{\gamma} N^{\delta} \\
& =R^{-5}\left(B \tilde{X}_{i} \tilde{X}_{j}+O(\Psi R)\right) .
\end{aligned}
$$

It follows that $\lim _{R \rightarrow 0} R^{-5} \Psi$ exists, and

$$
\lim _{R \rightarrow 0} \tilde{E}_{i j}=M \tilde{X}_{i} \tilde{X}_{j}
$$

where $M=\lim _{R \rightarrow 0} R^{-5} \Re \Psi$. Up to a multiplicative factor, $M$ is the integrand for the asymptotically defined, Ashtekar-Magnon global charges [4,5]. If $M$ is zero on $\mathscr{I}$ then all global charges are zero. $M$ is bounded on $\mathscr{I}$ away from the zeroes of $X$, but could be singular where $X$ is zero.

Now from (3.50)

$$
X^{\alpha} \partial_{\alpha}\left(M R^{5}\right)=0
$$

so with $X$ given by (3.55), (3.74) and (3.75), we need on $\mathscr{I}$ that

$$
B_{0}^{i} \frac{\partial M}{\partial x^{i}}-5 \sin \theta \cos (\phi-t) M=0 .
$$

On the equator (3.79) can be integrated to give

$$
M(t, \theta=\pi / 2, \phi)=(1-\sin (\phi-t))^{-5 / 2} f(\phi+t),
$$

for some function $f$ of $\phi+t$. From (3.78) one then has

$$
\tilde{E}_{t t}=(1-\sin (\phi-t))^{-1 / 2} f(\phi+t),
$$

so $f$ vanishes if a smooth global $\mathscr{I}$ exists.

For $\cos \theta \neq 0$ introduce $F$ by

$$
M=(\cos \theta)^{-5} F(t, \theta, \phi)
$$


then (3.79) becomes

$$
(-1+\sin \theta \sin (\phi-t)) \frac{\partial F}{\partial t}+\cos \theta \cos (\phi-t) \frac{\partial F}{\partial \theta}+(1-\sin (\phi-t) \csc \theta) \frac{\partial F}{\partial \phi}=0 .
$$

i.e. $F$ is constant on the integral curves of the vector field $B_{0}$, which we need to consider. We shall find that $\cos \theta$ is either zero or asymptotic to zero along every integral curve, and that the components of $X$ are asymptotic to zero along every integral curve. From the rate at which these quantities vanish, it will follow from (3.78) and (3.81) that, on the curves with $\cos \theta \neq 0, F$ vanishes on $\mathscr{I}$, and so does $M$, while it will follow from (3.80) that $M$ is zero on the curves with $\cos \theta=0$. Note that the Ashtekar-Magnon mass equals the boundary term which arises in Witten's positive energy argument by [27]. Hence, under the hypotheses of Theorem 3.9, we can then conclude that the initial data set can be embedded into anti-de Sitter space-time.

The integral curves of $B_{0}$ satisfy the system of equations

$$
\begin{aligned}
& \frac{d t}{d \lambda}=(-1+\sin \theta \sin (\phi-t)) \\
& \frac{d \theta}{d \lambda}=\cos \theta \cos (\phi-t) \\
& \frac{d \phi}{d \lambda}=(1-\sin (\phi-t) \csc \theta)
\end{aligned}
$$

in terms of a real parameter $\lambda$ along the curves.

Equating the right-hand-sides to zero, we see that the fixed points of $B_{0}$ lie on the curve $\Gamma$ defined by $\theta=\pi / 2, \phi-t=2 k \pi+\pi / 2$, for integer $k$, which is a helix on $\mathscr{I}$.

We first investigate integral curves with constant $\theta$. Any curve on which $\theta$ is constant must, by (3.83), have $\cos \theta$ or $\cos (\phi-t)$ vanishing, but in the second case, by (3.82) and (3.84), we arrive again at $\cos \theta=0$. Thus the only integral curves with $\theta$ constant have $\theta=\pi / 2$. Going further with these, we find that $\phi+t$ must be constant on them. Introduce $\gamma$ by $\phi-t=2 \gamma+\pi / 2$ then we find an equation for $\gamma$ which integrates to give

$$
\cot \gamma=2\left(\lambda_{0}-\lambda\right)
$$

for some constant $\lambda_{0}$, and so

$$
1-\sin (\phi-t)=2 \sin ^{2} \gamma=2\left(1+4\left(\lambda_{0}-\lambda\right)^{2}\right)^{-1} .
$$

Now suppose we have an integral curve with a point where $\cos \theta \neq 0$. It is straightforward to check that the following are constants along the integral curve:

$$
\begin{aligned}
a & :=\frac{\sin \theta \sin \phi-\cos t}{\cos \theta} \\
b & :=\frac{\sin \theta \cos \phi+\sin t}{\cos \theta}
\end{aligned}
$$


and then that

$$
\begin{aligned}
& \frac{d}{d \lambda}\left(\frac{\sin t}{\cos \theta}\right)=a \\
& \frac{d}{d \lambda}\left(\frac{\cos t}{\cos \theta}\right)=b
\end{aligned}
$$

so that

$$
\begin{aligned}
& \frac{\sin t}{\cos \theta}=a \lambda+c \\
& \frac{\cos t}{\cos \theta}=b \lambda+d,
\end{aligned}
$$

for constants $c$ and $d$. Squaring and adding these we find

$$
\sec ^{2} \theta=\left(a^{2}+b^{2}\right) \lambda^{2}+2(a c+b d) \lambda+\left(c^{2}+d^{2}\right) .
$$

If $a^{2}+b^{2}=0$ then $\theta$ would be constant, but we have just seen that the only integral curves with constant $\theta$ have $\cos \theta=0$ at all points. Thus $a^{2}+b^{2} \neq 0$, but now as $\lambda$ goes to plus or minus infinity, $\sec \theta$ is unbounded, so that $\theta$ must tend to $\pi / 2$ on each integral curve on which $\cos \theta$ is not always zero. More precisely, (3.89) shows that

$$
\theta-\pi / 2 \sim \lambda^{-1} \Longleftrightarrow \cos \theta \sim \lambda^{-1}
$$

at infinity, in the sense that there exists a constant $C$ such that $C^{-1} \lambda^{-1} \leq$ $\cos \theta \leq C \lambda^{-1}$.

We now look at the components of $B_{0}$ from (3.75) along the integral curve. From (3.85), (3.86), (3.87) and (3.88) we have

$$
\tan \theta \cos \phi=-a \lambda+(b-c) ; \quad \tan \theta \sin \phi=b \lambda+(a+d)
$$

so that

$$
\sin \theta \sin (\phi-t)-1=(a d-b c) \cos ^{2} \theta \sim \lambda^{-2} .
$$

To justify " ", we note that the cofficient of $\lambda^{-2}$ cannot be zero as

$$
a d-b c=\frac{a \cos t-b \sin t}{\cos \theta}
$$

and if this were zero then $t$ would be constant along the integral curve, which is inconsistent with (3.82). In the same manner we find that

$$
\begin{aligned}
\cos \theta \cos (\phi-t) & \sim \lambda^{-2}, \\
\frac{\sin (\phi-t)}{\sin \theta}-1 & \sim \lambda^{-2},
\end{aligned}
$$

so that, by (3.75), all components of $B_{0}$ vanish at the rate $O\left(\lambda^{-2}\right)$ and no faster along the integral curve. Putting this with (3.89) we find that $(\cos \theta)^{-5} X \otimes X$ is $O(\lambda)$ along the integral curve and therefore $F$, which is constant along the integral curve, must vanish. Thus $F$ vanishes on $\mathscr{I}$, therefore so does $M$ and all the asymptotically-defined momenta. 


\section{Ricci-flat conformal infinity}

So far we have been mostly assuming that Scri has spherical cross-sections. In this section we collect some results about alternatives. In section 4.1 we will prove an analogue of the angular-momentum inequality (3.2) for toroidal Scris; section 4.2 discusses some other possibilities. In the remaining two sections we review some examples saturating the inequality.

\subsection{Toroidal infinity}

We suppose that conformal infinity has toroidal topology

$$
\mathbb{T}^{n-1}:=S^{1} \times \ldots \times S^{1}
$$

with a flat metric $\check{h}$. The space-time metric

$$
{ }^{n+1} b=-\frac{r^{2}}{\ell^{2}} d t^{2}+\frac{\ell^{2}}{r^{2}} d r^{2}+r^{2} \check{h},
$$

where $\ell$ is related to the cosmological constant $\Lambda$ by the formula $2 \Lambda \ell^{2}=$ $-n(n-1)$, provides a static vacuum example satisfying all the conditions of the positivity theorem. The slices $t=$ const. have complete induced metric, with one conformally compactifiable end where $r \rightarrow \infty$, as well as a "cuspidal end" where $r \rightarrow 0$. The toroidal Kottler black holes [32] also belong to this class. Note that the coordinate $r$ in (4.1) can be rescaled by a constant factor, a subsequent redefinition of $\breve{h}$ and of $t$ preserves then the general form of the metric. A natural way of getting rid of this freedom is to assume that the volume of $\left(\mathbb{T}^{n-1}, \check{h}\right)$ equals $16 \pi$. Alternatively, one can assume that this volume equals one, and remove the normalisation constant $1 / 16 \pi$ in front of (2.2).

We consider the following, trivial spin structure over $\mathbb{T}^{n-1}$ : Let $\mathfrak{S}^{\prime \prime}$ be a product Hermitian bundle of spinors over $\mathbb{T}^{n-1}$, with a representation of the Clifford algebra of $\left(\mathbb{T}^{n-1}, \check{h}\right)$ via anti-Hermitian matrices. On $\mathbb{T}^{n-1}$ we use manifestly flat local coordinates $x^{a}, a=1, \ldots, n-1$, ranging from 0 to $2 \pi$, and we choose a spin frame so that all connection coefficients vanish, with the Clifford action of parallel vectors represented by constant matrices.

The Witten-type proof of the positive energy theorem requires imaginary Killing spinors in the asymptotic region $\mathscr{S}_{\text {ext }}$ "near conformal infinity"; in the current case this is the region $r \geq r_{0}$ for some large $r_{0}$, with the initial data metric $g$ approaching the space-part of (4.1) as in (2.1), and with $K_{i j}$ approaching $\stackrel{\circ}{K}_{i j}=0$ as required there. To construct those spinors we first consider $\mathfrak{S}^{\prime}=\mathfrak{S}^{\prime \prime} \oplus \mathfrak{S}^{\prime \prime}$, the direct sum of two copies of $\mathfrak{S}^{\prime \prime}$, equipped with the directsum sesquilinear product $\langle\cdot, \cdot\rangle_{\oplus}$ :

$$
\left\langle\left(\psi_{1}, \psi_{2}\right),\left(\varphi_{1}, \varphi_{2}\right)\right\rangle_{\oplus}:=\left\langle\psi_{1}, \varphi_{1}\right\rangle+\left\langle\psi_{2}, \varphi_{2}\right\rangle .
$$

For $X \in T \mathbb{T}^{n-1}$ we let $X$. denote the Clifford action of $X$ and, similarly to (2.11), for $\psi_{1}, \psi_{2} \in \mathfrak{S}^{\prime \prime}$ we set

$$
\gamma^{0}\left(\psi_{1}, \psi_{2}\right):=\left(\psi_{2}, \psi_{1}\right)
$$




$$
\begin{aligned}
& X \cdot\left(\psi_{1}, \psi_{2}\right):=\left(X \cdot \psi_{1},-X \cdot \psi_{2}\right), \\
& D_{X}\left(\psi_{1}, \psi_{2}\right):=\left(D_{X} \psi_{1}, D_{X} \psi_{2}\right) .
\end{aligned}
$$

One checks that $(4.3 \mathrm{~b})$ defines a representation of the Clifford algebra of $\left(\mathbb{T}^{n-1}, \check{h}\right)$ on $\mathfrak{S}^{\prime}$. Further

$$
\begin{aligned}
& \left(\gamma^{0}\right)^{2}=1, \\
& \forall X \in T \mathbb{T}^{n-1} \quad \gamma^{0} X \cdot=-X \cdot \gamma^{0}, \\
& \left(\gamma^{0}\right)^{\dagger}=\gamma^{0}, \\
& D \gamma^{0}=\gamma^{0} D,
\end{aligned}
$$

Next, it is convenient to pass to yet another direct sum bundle $\mathfrak{S}=\mathfrak{S}^{\prime} \oplus \mathfrak{S}^{\prime}$, equipped with the direct-sum Hermitian product which will be denoted by $\langle\cdot, \cdot\rangle_{\oplus \oplus}$. We define, for $\psi_{1}, \psi_{2} \in \mathfrak{S}^{\prime}, X \in T^{n-1} M$ and $a \in \mathbb{C}$,

$$
\begin{aligned}
& \gamma^{n}\left(\psi_{1}, \psi_{2}\right):=\left(-\psi_{2}, \psi_{1}\right) \\
& \left(X \cdot+a \gamma^{0}\right)\left(\psi_{1}, \psi_{2}\right):=\left(\left(X \cdot+a \gamma^{0}\right) \psi_{1},-\left(X \cdot+a \gamma^{0}\right) \psi_{2}\right), \\
& D_{X}\left(\psi_{1}, \psi_{2}\right):=\left(D_{X} \psi_{1}, D_{X} \psi_{2}\right) .
\end{aligned}
$$

This provides one more representation ${ }^{21}$ of the Clifford algebra of $\left(\mathbb{T}^{n-1}, \check{h}\right)$, on $\mathfrak{S}$, with moreover

$$
\begin{aligned}
& \left(\gamma^{n}\right)^{2}=-1, \\
& \forall X \in T \mathbb{T}^{n-1}, a \in \mathbb{C} \quad \gamma^{n}\left(X \cdot+a \gamma^{0}\right)=-\left(X \cdot+a \gamma^{0}\right) \gamma^{n}, \\
& \left(\gamma^{n}\right)^{\dagger}=-\gamma^{n}, \\
& D \gamma^{n}=\gamma^{n} D .
\end{aligned}
$$

We assume that on $\mathscr{S}_{\text {ext }}$ the background metric $b$ takes the form

$$
b=\left(d x^{n}\right)^{2}+e^{4 \mu x^{n}} \check{h} ;
$$

this corresponds to the space-part of the metric (4.1) when $\mu=1 /(2 \ell)$. The conformal boundary at infinity is constructed by multiplying by $e^{-4 \mu x^{n}}$, and replacing $x^{n}$ by $y=e^{-2 \mu x^{n}}$; the boundary is then the set $\{y=0\}$. We note that (4.7) is a complete space-form metric.

Any vector $Y \in T \mathscr{S}_{\text {ext }}$ can be written in form $Y=Y^{n} \partial_{n}+X^{a} e_{a}$, where $e_{a}=e^{-2 \mu x^{n}} f_{a}$, and where the $f_{a}$ 's form a $\breve{h}$-ON basis. Note that $\left\{\partial_{n}, e_{a}\right\}$ form a $b$-ON basis. We define the $b$-Clifford action of $Y$ on $\mathfrak{S}$ as

$$
Y \cdot=Y^{n} \gamma^{n}+X^{a} f_{a} \cdot
$$

Let the co-frame $\theta^{i}=\left(d x^{n}, \theta^{a}\right)$ be dual to $\left(\partial_{n}, e_{a}\right)$, then the only nonvanishing connection coefficients are $-\omega_{a n b}=\omega_{n a b}=-2 \mu \check{h}_{a b}$. One then has

$$
\stackrel{\circ}{D}_{k}=\partial_{k}-\frac{1}{4} \omega_{i j k} e^{i} \cdot e^{j} \cdot= \begin{cases}\partial_{n}, & k=n \\ \partial_{b}+\mu \gamma^{n} e_{b} \cdot & k=b .\end{cases}
$$

\footnotetext{
${ }^{21}$ This representation will not be irreducible, but this is irrelevant for the positivity argument. In fact, already the doubling (4.3) will lead to a reducible representation of the $\left(\mathbb{T}^{n-1}, \breve{h}\right)$-Clifford algebra extended by adding $\gamma^{0}$ when $n$ is odd.
} 
It follows (compare [7]) that for any $\chi \in \mathfrak{S}^{\prime}$, with constant entries, the spinor field

$$
\psi:=\frac{e^{\mu x^{n}}}{\sqrt{2}}(i \chi, \chi)
$$

defined over $\mathscr{S}_{\text {ext }}$, is an imaginary Killing spinor for $b$; by definition,

$$
\stackrel{\circ}{D}_{Y} \psi=-\mu i Y \cdot \psi
$$

where $\stackrel{\circ}{D}$ denotes the covariant derivative operator of $b$. One also has

$$
\forall Z \in T \mathscr{S}_{\text {ext }} \quad \stackrel{\circ}{\nabla}_{Z} \hat{\psi}:=\left(\stackrel{\circ}{D}_{Z}-\frac{1}{2} \stackrel{\circ}{K}_{i}^{j} Z^{i} e_{j} \cdot \gamma^{0}\right) \hat{\psi}=-\mu i Z \cdot \hat{\psi}
$$

because the background extrinsic curvature $\stackrel{\circ}{K}_{i j}$ of the slices $t=0$ for the associated space-time background metric ${ }^{n+1} b$ vanishes.

Let $\mathscr{K}$ denote the space of imaginary Killing spinors $\psi \in \Gamma \mathfrak{S}$ constructed so far. As already mentioned in Section 2, to any element of $\mathscr{K}$ one can associate a KID of the background initial data $(b, 0)$ as follows

$$
\mathscr{K} \ni \psi \rightarrow\left(V=\langle\psi, \psi\rangle_{\oplus \oplus}, Y=\left\langle\psi, \gamma^{n} \cdot \gamma^{0} \psi\right\rangle_{\oplus \oplus} \partial_{n}+\sum_{a}\left\langle\psi, f_{a} \cdot \gamma^{0} \psi\right\rangle_{\oplus \oplus} e_{a}\right) .
$$

Chasing through the definitions we find

$$
\begin{aligned}
V & =\langle\psi, \psi\rangle_{\oplus \oplus}=e^{2 \mu x^{n}}\langle\chi, \underbrace{\chi}_{=\left(\chi_{1}, \chi_{2}\right)}\rangle_{\oplus} \\
& =e^{2 \mu x^{n}}\left(\left\langle\chi_{1}, \chi_{1}\right\rangle+\left\langle\chi_{2}, \chi_{2}\right\rangle\right) \\
Y & =\underbrace{\left\langle\psi, \gamma^{n} \cdot \gamma^{0} \psi\right\rangle_{\oplus \oplus}}_{=0} \partial_{n}+\sum_{a}\left\langle\psi, f_{a} \cdot \gamma^{0} \psi\right\rangle_{\oplus \oplus} e_{a} \\
& =e^{2 \mu x^{n}} \sum_{a}\left\langle\chi, f_{a} \cdot \gamma^{0} \chi\right\rangle_{\oplus} e_{a}=\sum_{a}\left(\left\langle\chi_{1}, f_{a} \cdot \chi_{2}\right\rangle-\left\langle\chi_{2}, f_{a} \cdot \chi_{1}\right\rangle\right) f_{a} \\
& =2 \sum_{a} \Re\left(\left\langle\chi_{1}, f_{a} \cdot \chi_{2}\right\rangle\right) f_{a} .
\end{aligned}
$$

Let $m$ denote the value of $H$ corresponding to the background-KID $V=$ $e^{2 \mu x^{n}} / \ell, Y=0$. This last KID corresponds to the Killing vector $\partial_{t}$ of the metric (4.1), so that $m$ has the interpretation as energy. Similarly let $j_{(b)}$ be the value of $H$ corresponding to $f_{b}$; thus $V=0$ and $Y^{a} \partial_{a}=f_{b}$. Clearly each $j_{(b)}$ has a natural interpretation of angular momentum.

Under the hypotheses of Theorem 2.1, one concludes that the composition of (4.11) with the Hamiltonian map (2.2) defines a positive Hermitian form on $\mathscr{K}$. We have

$$
\begin{aligned}
H(V, Y) & =H\left(\left(\left\langle\chi_{1}, \chi_{1}\right\rangle+\left\langle\chi_{2}, \chi_{2}\right\rangle\right)\left(e^{2 \mu x^{n}}, 0\right)+2 \sum_{a} \Re\left(\left\langle\chi_{1}, f_{a} \cdot \chi_{2}\right\rangle\right)\left(0, f_{a}\right)\right) \\
& =\left(\left\langle\chi_{1}, \chi_{1}\right\rangle+\left\langle\chi_{2}, \chi_{2}\right\rangle\right) \ell m+2 \sum_{a} \Re\left(\left\langle\chi_{1}, f_{a} \cdot \chi_{2}\right\rangle\right) j_{(a)} \geq 0,
\end{aligned}
$$


for all constant spinors $\left(\chi_{1}, \chi_{2}\right)$. This is possible if and only if ${ }^{22}$

$$
m \geq \sqrt{-\frac{2 \Lambda}{n(n-1)}}|\vec{j}|, \quad|\vec{j}|:=\sqrt{j_{(1)}^{2}+\ldots+j_{(n)}^{2}} .
$$

We have thus derived the toroidal equivalent of Maerten's inequality (3.2); we emphasise the spin-structure compatibility condition (2.7).

In space-dimension three (4.14) can be viewed as the special case $\vec{c}=0$ of (3.2), but the justification of this appears to require the work above.

Let $j_{a}$ be the angular momentum associated with the Killing vector $\partial_{a}$. It should be clear that with this definition the inequality in (4.14) remains valid if $|\vec{j}|$ is taken to be $\sqrt{h^{a b} j_{a} j_{b}}$, where $\check{h}^{a b}$ is the inverse matrix to $\check{h}_{a b}:=\check{h}\left(\partial_{a}, \partial_{b}\right)$.

\subsection{General conformal infinities with parallel spinors}

We now consider a metric (4.1), without assuming that $\check{h}$ is flat: instead we assume that the manifold $\left({ }^{n-1} M, \check{h}\right)$ carries a non-trivial covariantly constant spinor $\chi$, section of a spinor bundle $\mathfrak{S}^{\prime \prime}$. (Such manifolds are necessarily Ricci flat, compare $[9,12,30,39,40]$.) The construction of the background imaginary Killing spinors of the previous section carries over with only trivial modifications to such a setting. Under the hypotheses of Theorem 2.1 we then obtain a positive definite quadratic functional on the space of covariantly constant spinors of $\left({ }^{n-1} M, \check{h}\right)$. It appears that an optimal form of the resulting constraints has to be analysed case-by-case. Here we only note the following: For every $\check{h}$-parallel $\chi$ the norm squared $\langle\chi, \chi\rangle$ is constant over ${ }^{n-1} M$. It follows that we can normalise $\chi$ to obtain two KIDs as in (4.11) with $\chi_{2}= \pm \chi_{1}=\chi$ in (4.12)-(4.13), and with time component of the associated KIDs equal to one. The positivity of $H$ for both the plus and minus signs then gives

$$
\ell m \geq|j|,
$$

where $j$ is the angular momentum associated with the $b$-Killing vector $Y$ corresponding to $\chi$, and $\ell$ has been defined in (3.9). We thus obtain positivity of $m$, together with an upper bound on $|j|$ in terms of $m$. The result is optimal if the space of covariantly constant spinors of $\left({ }^{n-1} M, \check{h}\right)$ is one-dimensional. Otherwise we clearly also have the non-optimal inequality

$$
\ell m \geq \sup _{\psi}\left|j\left(X_{\psi}\right)\right|
$$

where the supremum is taken over the covariantly Killing spinors $\psi$ normalised as described above, and $j\left(X_{\psi}\right)$ denotes the angular momentum along the Killing vector $X_{\psi}$ associated to $\psi$.

\footnotetext{
${ }^{22}$ Indeed, if $|\vec{j}|=0$ the inequality (4.14) is clear. Otherwise choose $\chi_{2}=\sum_{a} j_{(a)} f_{a} \cdot \chi_{1} /|\vec{J}|$ to conclude that (4.14) is necessary. The proof of sufficiency is left to the reader.
} 


\subsection{Nonrigidity in the toroidal case for $n=3$}

By Section 3.1 equality in (4.14) leads, locally, to space-forms or to Siklos waves. In order to see that those are compatible with the toroidal topology at infinity note, first, that the metric (3.45) with $2 b^{2}=1$ and $H=0$ gives anti-de Sitter, by introducing $t=(u+v) / \sqrt{2}$ and $z=(v-u) / \sqrt{2}$ :

$$
g=\frac{1}{x^{2}}\left(d x^{2}+d y^{2}+d z^{2}-d t^{2}\right) .
$$

This metric covers part of anti-de Sitter space-time. However, we now impose a periodic identification in $y$ and in $z$. Then this is a metric with a $\mathscr{I}$ which is topologically $\mathbb{T}^{2} \times \mathbb{R}$ at $x=0$ and a 'hyperbolic cusp' as $x \rightarrow \infty$, as in (4.1). We can retain these asymptotics with a nonzero $H(u, x, y)$ which is suitably periodic in $u$ and $y$ and decays appropriately in $x$ as $x$ goes to zero. A simple class of examples may be generated as follows: take

$$
H=x f^{\prime}(x)-f(x),
$$

then from (3.48) we find

$$
\Phi=-\frac{x^{6}}{4}\left(\frac{f^{\prime \prime}}{x}\right)^{\prime}
$$

while from (3.49)

$$
\Psi=-\frac{x^{4}}{4}\left(x f^{\prime \prime}\right)^{\prime}
$$

For the dominant energy condition $\left(T_{a b} v^{a} v^{b} \geq 0\right.$ for timelike $\left.v^{a}\right)$ to hold we need $\Phi$ to be non-negative so set $\Phi=\frac{x^{6}}{4} \rho(x)$ for a non-negative function $\rho$. For simplicity, we assume that $\rho$ has compact support, and then we solve for $f^{\prime \prime}$ as

$$
f^{\prime \prime}(x)=x \int_{x}^{\infty} \rho(y) d y .
$$

Suppose $\rho$ is supported in $0<a<x<b<\infty$, then so is $\Phi$ (and so also is the energy-momentum tensor). For $x<a$, we find $f^{\prime \prime}=m x$ with $m=\int_{a}^{b} \rho d x$ and then $\Psi=-m x^{5} / 2$, which is the rate of decay we found we required in (3.4). Thus $\mathscr{I}$ exists at $x=0$ with this $H$, as with $H=0$. Letting $t$ and $z$ be as at the beginning of this section, we require the level sets of $t$ to be spacelike. This is equivalent to

$$
H<2,
$$

which can be arranged by the choice of $f(0)$ for any $\rho$ as above. We note the following formulae for the metric and second fundamental form of the hypersurface $\mathscr{S}:=\{t=0\}$

$$
\begin{gathered}
g_{i j} d x^{i} d x^{j}=x^{-2}\left\{d x^{2}+d y^{2}+\left(1-\frac{H}{2}\right) d z^{2}\right\}, \quad \sqrt{\operatorname{det} g_{i j}}=x^{-3} \sqrt{1-\frac{H}{2}}, \\
g_{t t}=-x^{-2}\left(1+\frac{H}{2}\right), \quad g_{z t}=x^{-2} \frac{H}{2}, \\
K_{i j} d x^{i} d x^{j}=-\frac{H^{\prime}}{x \sqrt{4-2 H}} d x d z \\
|K|_{g}^{2}=\frac{\left(x H^{\prime}\right)^{2}}{2(2-H)^{2}},
\end{gathered}
$$


which shows that $K$ satisfies the decay conditions needed for a well-defined mass (recall that $\rho$ vanishes near $x=0$ ). One can check that

$$
H(x)=H(0)+\int_{0}^{x} \frac{y^{3}}{3} \rho(y) d y+\frac{x^{3}}{3} \int_{x}^{\infty} \rho(y) d y,
$$

so that $H$ is a non-decreasing function of $x$ for non-negative $\rho$ and, subsequently, that (4.18) will hold if and only if

$$
3 H(0)+\int_{0}^{\infty} x^{3} \rho(x) d x<6 .
$$

Assuming this condition, and a compact support of $\rho$ in $(0, \infty)$, the hypersurface $\mathscr{S}=\{t=0\}$ with the induced fields provides an example of non-trivial initial data set which saturates the inequality (4.14), and satisfies all the hypotheses of the positive energy theorem in Section 2.

For $x>b, \Psi=0$ and the space-time is locally anti-de Sitter. For example, if we choose $H(0)=0=f(0)$ (note that the choice of $f^{\prime}(0)$ is irrelevant as it does not change $H$ ) then for $x>b, H$ is constant and equal to $H_{\infty}=\left(\int_{a}^{b} x^{3} \rho d x\right) / 3$. The metric is

$$
g=\frac{1}{x^{2}}\left(d x^{2}+d y^{2}+d z^{2}-d t^{2}-\frac{H_{\infty}}{2}(d t-d z)^{2}\right)
$$

but the coordinate transformation

$$
\begin{aligned}
d \tilde{z} & =d z\left(1-\frac{H_{\infty}}{4}\right)+\frac{H_{\infty}}{4} d t \\
d \tilde{t} & =-\frac{H_{\infty}}{4} d z+\left(1+\frac{H_{\infty}}{4}\right) d t
\end{aligned}
$$

has the effect of setting $H_{\infty}$ to zero.

Another interesting example is $f=C \sinh x \sin y \sin u$, where $C$ is a constant. Now $H=x f_{x}-f$ gives vacuum. It satisfies the asymptotic conditions, with $\Psi=O\left(x^{5}\right)$ for small $x$, but because the solution is exponentially large for large $x$ the existence of globally regular spacelike surfaces is not clear. This leads naturally to the question of existence of non-trivial vacuum initial data sets saturating the angular momentum inequality (4.14). Recall that no such black hole solutions exist by the results in Section 3.2, but the general result is not known.

\subsection{Higher dimensional examples}

Gibbons and Ruback [23] have presented some metrics which are generalisations of the Siklos metrics to higher dimensions (compare [8, p. 14]). In space dimension $n$ (so space-time dimension $(n+1)$ ), the metrics can be written in the form

$$
g^{G R}=\frac{1}{2 b^{2} x^{2}}\left(d x^{2}+h_{a b} d y^{a} d y^{b}-2 d u d v-H\left(u, x, y^{a}\right) d u^{2}\right) .
$$


where $h=h_{a b} d y^{a} d y^{b}$ is a Ricci-flat, Riemannian metric on an $(n-2)$-dimensional manifold ${ }^{n-2} M$ (compare (3.45)). From now on we set $2 b^{2}=1$. To analyse the imaginary Killing spinor equation we use the frame

$$
\theta^{0}=\frac{d u}{x}, \quad \theta^{1}=\frac{1}{x}\left(d v+\frac{H}{2} d u\right), \quad \theta^{2}=\frac{d x}{x}, \quad \theta^{a}=\frac{1}{x} \check{\theta}^{a},
$$

where $\check{\theta}^{a}$ is an ON-frame for $\left({ }^{n-2} M, h\right)$. A somewhat lengthy calculation shows that if $\psi_{h}$ is a covariantly constant spinor for $h$ then, in a basis of the spinor bundle where the $\gamma$-matrices are independent of $x, u$ and $v$, the spinor field $\psi=x^{-1 / 2} \psi_{h}$ is an imaginary Killing spinor for (4.24) and, in fact:

Proposition 4.1 The metrics (4.24) admit non-trivial imaginary Killing spinors if and only if $\left({ }^{n-2} M, h\right)$ admits non-zero covariantly constant spinors.

So, when such spinors exist, the volume integral in the Witten identity vanishes, therefore so does the boundary integral. Assuming the asymptotic conditions permit the existence of $\mathscr{I}$, the metrics (4.24) will therefore saturate the $n$-dimensional version of our bounds.

The Ricci tensor Ric ${ }^{G R}$ for $g^{G R}$ may be written

$$
\operatorname{Ric}^{G R}=-n g^{G R}+2 \Phi X \otimes X
$$

where $X=\partial / \partial v$ and $\Phi$ is the function

$$
\Phi=-\frac{x^{4}}{4}\left(\frac{\partial^{2} H}{\partial x^{2}}-\frac{(n-1)}{x} \frac{\partial H}{\partial x}+\Delta_{h} H\right)
$$

where $\Delta_{h}$ is the Laplacian for $h$. The dominant energy condition again requires $\Phi$ to be positive and as in the three dimensional case we can readily find solutions with $H$ independent of $u$ and $y^{a}$ : set $\Phi=\frac{1}{8} x^{n+3} \rho(x)$ then solve

$$
\left(x^{1-n} H^{\prime}(x)\right)^{\prime}=-\rho,
$$

where prime denotes $d / d x$, to find

$$
H^{\prime}(x)=x^{n-1} \int_{x}^{\infty} \rho(s) d s,
$$

(compare (4.17)). Now if we assume that ${ }^{n-2} M$ is compact and that $u$ is periodic, we obtain a solution with a $\mathscr{I}$ located at $x=0$, whose cross-sections are ${ }^{n-2} M \times S^{1}$. The discussion around (4.18) goes through as before: $K_{i j} d x^{i} d x^{j}$ is as in (4.21), where now $x^{i}=\left(x, y^{a}, z\right)$, and (4.19) is replaced by

$$
g_{i j} d x^{i} d x^{j}=x^{-2}\left\{d x^{2}+h_{a b} d y^{a} d y^{b}+\left(1-\frac{H}{2}\right) d z^{2}\right\} .
$$

Therefore, under (4.18), and assuming that $\rho$ is non-negative and compactly supported, these solutions will satisfy the global and asymptotic conditions of the positivity theorem. 
For the counterpart of (3.78) we obtain, with conventions as above and in [4] and with $R=\frac{x}{\sqrt{2}}$

$$
\begin{aligned}
\tilde{E}_{i j} & =\frac{1}{(n-2)}\left(\frac{x}{\sqrt{2}}\right)^{2-n} W_{i \alpha j \beta} N^{\alpha} N^{\beta} \\
& =\frac{-2}{(n-1)}\left(\frac{x}{\sqrt{2}}\right)^{2-n} H^{\prime \prime} \tilde{X}_{i} \tilde{X}_{j}
\end{aligned}
$$

which, by (4.25), has a finite limit on $\mathscr{I}$ where $x=0$.

The imaginary Killing spinors described immediately before the statement of Proposition 4.1 have the property that

$$
X \cdot \psi=0,
$$

where, as before, $X=\left\langle\psi, \gamma^{0} \gamma^{\mu} \psi\right\rangle \partial_{\mu}$, and $\cdot$ denotes the space-time Clifford multiplication. An analysis similar to that in Section 3.2 applies, whatever $n \geq 3$, as follows: Differentiating (4.26) one finds, for all $Y$,

$$
\left(\nabla_{Y} X\right) \cdot \psi \sim \psi
$$

By e.g. [34, Lemma 2.1, point 2] we then have

$$
\nabla_{Y} X \sim X
$$

which immediately implies staticity:

$$
X_{[\mu} \nabla_{\nu} X_{\rho]}=0 .
$$

The search for black hole solutions in this class is inconclusive: Any event horizon would have to be degenerate, and then the remaining arguments of Section 3.2 show that the space-metric on the event horizon has a Ricci tensor proportional to the metric, with negative proportionality constant. The consequences of this are not clear, as the constraints imposed by topological censorship [20] are less stringent in higher dimensions.

Acknowledgements: We thank the Newton Institute, Cambridge (PTC, DM, PT), and the AEI, Golm (PTC, PT), for hospitality and support during part of work on this paper. We are grateful to H. Baum, G. Gibbons, F. Leitner, and M. Stern for comments and bibliographical advice, and to M-G. Greuel and S. Szybka for help with Singular and MAPle calculations.

\section{References}

[1] M.T. Anderson, On stationary vacuum solutions to the Einstein equations, Annales H. Poincaré 1 (2000), 977-994, gr-qc/0001091.

[2] M.T. Anderson, P.T. Chruściel, and E. Delay, Non-trivial, static, geodesically complete space-times with a negative cosmological constant. II. $n \geq 5$, AdS/CFT correspondence: Einstein metrics and their conformal boundaries, IRMA Lect. Math. Theor. Phys., vol. 8, Eur. Math. Soc., Zürich, 2005, gr-qc/0401081, pp. 165-204. 
[3] L. Andersson and M. Dahl, Scalar curvature rigidity for asymptotically locally hyperbolic manifolds, Annals of Global Anal. and Geom. 16 (1998), 1-27, dg-ga/9707017.

[4] A. Ashtekar and S. Das, Asymptotically anti-de Sitter space-times: Conserved quantities, Class. Quantum Grav. 17 (2000), L17-L30, hepth/9911230. MR MR1739432 (2001g:83054)

[5] A. Ashtekar and A. Magnon, Asymptotically anti-de Sitter space-times, Class. Quantum Grav. 1 (1984), L39-L44. MR 85h:83029

[6] R. Bartnik and P.T. Chruściel, Boundary value problems for Dirac-type equations, (2003), math.DG/0307278.

[7] H. Baum, Complete Riemannian manifolds with imaginary Killing spinors, Ann. Global Anal. Geom. 7 (1989), 205-226. MR MR1039119 (91k:58130)

[8] — Twistor and Killing spinors in Lorentzian geometry, Global analysis and harmonic analysis (Marseille-Luminy, 1999), Sémin. Congr., vol. 4, Soc. Math. France, Paris, 2000, pp. 35-52. MR MR1822354 (2002d:53060)

[9] H. Baum and I. Kath, Parallel spinors and holonomy groups on pseudoRiemannian spin manifolds, Ann. Global Anal. Geom. 17 (1999), 1-17. MR MR1674339 (2000d:53076)

[10] D. Birmingham, Topological black holes in anti-de Sitter space, Class. Quantum Grav. 16 (1999), 1197-1205, hep-th/9808032.

[11] C. Bohle, Killing spinors on Lorentzian manifolds, Jour. Geom. Phys. 45 (2003), 285-308. MR MR1952661 (2004i:53056)

[12] R.L. Bryant, Pseudo-Riemannian metrics with parallel spinor fields and vanishing Ricci tensor, Global analysis and harmonic analysis (MarseilleLuminy, 1999), Sémin. Congr., vol. 4, Soc. Math. France, Paris, 2000, math.DG/0004073, pp. 53-94. MR MR1822355 (2002h:53082)

[13] P.T. Chruściel, On the relation between the Einstein and the Komar expressions for the energy of the gravitational field, Ann. Inst. H. Poincaré 42 (1985), 267-282.

[14] P.T. Chruściel and M. Herzlich, The mass of asymptotically hyperbolic Riemannian manifolds, Pacific Jour. Math. 212 (2003), 231-264, dgga/0110035.

[15] P.T. Chruściel, J. Jezierski, and S. Łȩski, The Trautman-Bondi mass of hyperboloidal initial data sets, Adv. Theor. Math. Phys. 8 (2004), 83-139, gr-qc/0307109.

[16] P.T. Chruściel and G. Nagy, The Hamiltonian mass of asymptotically antide Sitter space-times, Class. Quantum Grav. 18 (2001), L61-L68, hepth/0011270. 
[17] P.T. Chruściel, H.S. Reall, and K.P. Tod, On non-existence of static vacuum black holes with degenerate components of the event horizon, Class. Quantum Grav. 23 (2005), 549-554, gr-qc/0512041.

[18] R. Clarkson and R. B. Mann, Eguchi-Hanson solitons, (2005), hepth/0508109.

[19] M. Cvetič, P. Gao, and J. Simon, Supersymmetric Kerr-anti-de Sitter solutions, Phys. Rev. D72 (2005), 021701, hep-th/0504136.

[20] G.J. Galloway, K. Schleich, D.M. Witt, and E. Woolgar, Topological censorship and higher genus black holes, Phys. Rev. D60 (1999), 104039, grqc/9902061.

[21] G.W. Gibbons, S.W. Hawking, G.T. Horowitz, and M.J. Perry, Positive mass theorem for black holes, Commun. Math. Phys. 88 (1983), 295-308.

[22] G.W. Gibbons, C.M. Hull, and N.P. Warner, The stability of gauged supergravity, Nucl. Phys. B218 (1983), 173-190.

[23] G.W. Gibbons and P.J. Ruback, Classical gravitons and their stability in higher dimensions, Phys. Lett. B171 (1986), 390-395.

[24] J.B. Gutowski and H.S. Reall, General supersymmetric AdS(5) black holes, JHEP 04 (2004), 048.

[25] M. Henneaux and C. Teitelboim, Asymptotically anti-de Sitter spaces, Commun. Math. Phys. 98 (1985), 391-424. MR 86f:83030

[26] M. Herzlich, The positive mass theorem for black holes revisited, Jour. Geom. Phys. 26 (1998), 97-111.

[27] S. Hollands, A. Ishibashi, and D. Marolf, Comparison between various notions of conserved charges in asymptotically AdS spacetimes, Class. Quantum Grav. 22 (2005), 2881-2920, hep-th/0503045. MR MR2154192

[28] G.T. Horowitz and R.C. Myers, The AdS/CFT correspondence and a new positive energy conjecture for general relativity, Phys. Rev. D59 (1999), 026005 (12 pp.), hep-th/9808079.

[29] S.A. Huggett and K.P. Tod, An introduction to twistor theory, second ed., London Mathematical Society Student Texts, vol. 4, Cambridge University Press, Cambridge, 1994. MR MR1292461 (95d:32036)

[30] A. Ikemakhen, Groupes d'holonomie et spineurs parallèles sur les variétés pseudo-riemanniennes complètement réductibles, C. R. Math. Acad. Sci. Paris 339 (2004), 203-208. MR MR2078075 (2005h:53076)

[31] I. Kath, Killing spinors on pseudo-Riemannian manifolds, 1999, Habilitationsschrift, Humboldt Universität.

[32] F. Kottler, Über die physikalischen Grundlagen der Einsteinschen Gravitationstheorie, Annalen der Physik 56 (1918), 401-462. 
[33] H. Blaine Lawson and M-L. Michelsohn, Spin geometry, Princeton Mathematical Series, vol. 38, Princeton University Press, Princeton, 1989.

[34] F. Leitner, Imaginary Killing spinors in Lorentzian geometry, Jour. Math. Phys. 44 (2003), 4795-4806. MR MR2008949 (2005b:53075)

[35] D. Maerten, Positive energy-momentum theorem in asymptotically Anti-de Sitter space-times, Annales H. Poincaré 7 (2006), 975-1011, math.DG/0506061.

[36] T. Parker and C. Taubes, On Witten's proof of the positive energy theorem, Commun. Math. Phys. 84 (1982), 223-238.

[37] R. Penrose and W. Rindler, Spinors and spacetime I: Two-spinor calculus and relativistic fields, Cambridge University Press, Cambridge, 1984.

[38] S.T.C. Siklos, Lobatchevski plane gravitational waves, Galaxies, axisymmetric systems and relativity: essays presented to W.B. Bonnor on his 65th birthday (M.A.H. MacCallum, ed.), Cambridge Univ. Press, Cambridge, UK, 1985, pp. 247-274.

[39] McKenzie Y. Wang, Parallel spinors and parallel forms, Ann. Global Anal. Geom. 7 (1989), 59-68. MR MR1029845 (91g:53053)

[40] _ On non-simply connected manifolds with non-trivial parallel spinors, Ann. Global Anal. Geom. 13 (1995), 31-42. MR MR1327109 (96a:53066)

[41] X. Wang, Mass for asymptotically hyperbolic manifolds, Jour. Diff. Geom. 57 (2001), 273-299.

[42] X. Zhang, A definition of total energy-momenta and the positive mass theorem on asymptotically hyperbolic 3 manifolds I, Commun. Math. Phys. 249 (2004), 529-548. 